\title{
Casimir forces in multilayer magnetodielectrics with both gain and loss
}

\author{
Amooghorban, Ehsan; Wubs, Martijn; Mortensen, N. Asger; Kheirandish, Fardin
}

Published in:

Physical Review A

Link to article, DOI:

10.1103/PhysRevA.84.013806

Publication date:

2011

Document Version

Publisher's PDF, also known as Version of record

Link back to DTU Orbit

Citation $(A P A)$ :

Amooghorban, E., Wubs, M., Mortensen, N. A., \& Kheirandish, F. (2011). Casimir forces in multilayer magnetodielectrics with both gain and loss. Physical Review A, 84(1), 013806.

https://doi.org/10.1103/PhysRevA.84.013806

\section{General rights}

Copyright and moral rights for the publications made accessible in the public portal are retained by the authors and/or other copyright owners and it is a condition of accessing publications that users recognise and abide by the legal requirements associated with these rights.

- Users may download and print one copy of any publication from the public portal for the purpose of private study or research.

- You may not further distribute the material or use it for any profit-making activity or commercial gain

- You may freely distribute the URL identifying the publication in the public portal 


\title{
Casimir forces in multilayer magnetodielectrics with both gain and loss
}

\author{
Ehsan Amooghorban, ${ }^{1,2, *}$ Martijn Wubs, ${ }^{1}$ N. Asger Mortensen, ${ }^{1}$ and Fardin Kheirandish ${ }^{2}$ \\ ${ }^{1}$ Department of Photonics Engineering, Technical University of Denmark, DK-2800 Kongens. Lyngby, Denmark \\ ${ }^{2}$ Department of Physics, University of Isfahan, Hezar Jarib Avenue, Isfahan, Iran
}

(Received 7 April 2011; published 7 July 2011)

\begin{abstract}
A path-integral approach to the quantization of the electromagnetic field in a linearly amplifying magnetodielectric medium is presented. Two continua of inverted harmonic oscillators are used to describe the polarizability and magnetizability of the amplifying medium. The causal susceptibilities of the amplifying medium, with negative imaginary parts in finite frequency intervals, are identified and their relationships to microscopic coupling functions are determined. By carefully relating the two-point functions of the field theory to the optical Green functions, we calculate the Casimir energy and Casimir forces for a multilayer magnetodielectric medium with both gain and loss. We point out the essential differences with a purely passive layered medium. For a single layer, we find different bounds on the Casimir force for fully amplifying and for lossy media. The force is attractive in both cases, even if the medium exhibits negative refraction. From our Lagrangian we also derive by canonical quantization the postulates of the phenomenological theory of amplifying magnetodielectrics.
\end{abstract}

DOI: 10.1103/PhysRevA.84.013806

PACS number(s): 42.50.Nn, 12.20.Ds, 03.70.+k

\section{INTRODUCTION}

The Casimir force is a pure quantum effect that can be considered as the macroscopic manifestation of the vacuum fluctuations of the electromagnetic fields in the presence of boundaries. Originally derived in 1948 for two ideally conducting or reflecting plates in a vacuum [1], the Casimir force per area $F_{\mathrm{C}} / A$ was found to be $-\hbar c \pi^{2} /\left(240 d^{4}\right)$, an attractive force with characteristic inverse fourth-power dependence on the plate separation $d$. A theory for Casimir forces between parallel dielectrics was developed by Lifshitz et al. [2], further refined by Schwinger et al. [3], and since then extended to arbitrary multilayer dielectrics [4-8] and other geometries.

In recent years, the Casimir force has become technologically relevant, with the development of micro- and nanoelectromechanical systems with small components at close proximity to each other. On the one hand, the Casimir force can be a major cause of stiction (i.e., microscopic components sticking together), friction, or adhesion and thus forms a possible obstacle for the operation of nanostructured devices. On the other hand, a novel class of microelectromechanical systems (MEMSs) works only because of the Casimir force $[9,10]$.

It is therefore both fundamentally interesting and technologically relevant to what extent the Casimir force can be controlled by changing the electromagnetic environment. For most geometries, the Casimir force between two media separated by a vacuum is an attractive force, with a magnitude that becomes appreciable in the submicron range and rapidly increases in the nanometer range. However, Lifschitz predicted that the Casimir force for parallel dielectric layers can be attractive or repulsive, depending on the relative values of the dielectric constants of the successive layers [2]. The first experimental confirmation of his prediction came only recently: Munday et al. found Casimir forces with different signs for suitably chosen interacting materials immersed in a

*amooghorban@sci.ui.ac.ir fluid [11], with a measured repulsive interaction which was weaker than the attractive counterpart. Further measurements of Casimir forces are reported in Refs. [12-21].

If Casimir forces could be made repulsive, then this could eliminate the unwanted phenomenon of stiction [22], enable quantum levitation of objects in a fluid, and lead to new classes of switchable nanoscale devices with ultralow static friction [23-26]. So what options exist to make the Casimir force repulsive, besides Lifschitz's suggestion?

Metamaterials have been proposed to this end. However, loss in metallic substructures may turn a repulsive force into an attractive one, which may explain why repulsive Casimir forces with metamaterials have not yet been reported [27,28]. Recently, Zhao et al. showed theoretically that a repulsive Casimir force could be realized with metamaterials with strong chirality [29]. Another mechanism to obtain repulsive Casimir forces is Boyer's Casimir repulsion based on an asymmetric three-layer setup of a nonmagnetic medium on the one end and a purely magnetic medium on the other, separated by a vacuum [30,31]. It relies on the nontrivial possibility of developing new artificial negative-index metamaterials [28].

A third option to obtain repulsive Casimir forces, and the one considered in this paper, is the use of media with optical gain $[32,33]$. Indeed, the main aim of the present paper is to determine Casimir energy and forces in amplifying magnetodielectrics. By amplifying medium, we mean a medium for which the imaginary part of the electric or the magnetic susceptibility becomes negative for one or more frequency intervals $\left(\operatorname{Im}[\varepsilon(\omega)] \equiv \varepsilon_{\mathrm{I}}(\omega)<0\right.$ or $\left.\mu_{\mathrm{I}}(\omega)<0\right)$, in contrast to lossy systems for which both $\varepsilon_{\mathrm{I}}(\omega)$ and $\mu_{\mathrm{I}}(\omega)$ are always positive. We allow for gain not only in the electric but also in the magnetic response, thereby treating electric and magnetic fields equally in our theory.

Since it is a quantum mechanical effect, the calculation of the Casimir force for media with gain requires a consistent procedure for quantization of the electromagnetic field in the presence of an amplifying medium. This can be done with the concept of inverted quantum harmonic oscillators introduced by Glauber [34]. Generally, a rigorous quantization procedure would require a Lagrangian and Hamiltonian formulation of 
the theory, followed by the standard canonical quantization rules. Currently, a consistent phenomenological approach exists to macroscopic quantum electrodynamics in presence of amplifying media, but no canonical formulation is attempted [35-39]. We derive the postulates of this phenomenological theory from a canonical quantum theory, where continua of inverted oscillators are used to describe linearly amplifying media.

The Casimir force in the presence of amplifying materials, with or without negative index, has begun to be explored only recently. Leonhardt and Philbin calculated the effects of an amplifying dielectric on the Casimir force, based on the assumption that the well-known Lifshitz formula for the Casimir force is applicable without change to amplifying media [33], and found that the Casimir force in the presence of this medium is repulsive. We address this issue here as well.

Sambale et al. [40,41] apply the phenomenological quantization of the electromagnetic field for amplifying magnetodielectric media to calculate the Casimir and Casimir-Polder forces. They find that the Casimir-Polder force on a weakly polarizable plate of excited gas atoms is attractive at short distances from a mirror and oscillates between attraction and repulsion for larger plate-mirror separations. However, at a more technical level, there is some controversy about the applicability of the Minkowski stress tensor, and an alternative, Lorentz-force-based tensor was proposed [42]. This approach was adopted in Refs. [40,41] to calculate the Casimir (-Polder) forces but is disputed by Pitaevskii [43] and Brevik [44].

Our approach to calculating Casimir forces is different. We first develop a path-integral method for the quantization of the electromagnetic field in linearly amplifying magnetodielectrics. We benefit from and generalize recent results obtained with path integrals. In Ref. [45], Li and Kardar developed a path-integral approach for computing fluctuationinduced forces between manifolds immersed in a correlated fluid. Golestanian and Kardar extended this formalism to arbitrary but small deformations of the boundaries and focused on the mechanical response of the vacuum [46]. Emig and colleagues also used the path-integral formalism to obtain the normal and lateral Casimir forces between two sinusoidally corrugated perfectly conducting surfaces [47]. Recently, one of the authors extended this formalism to calculate the Casimir force between two perfectly conducting plates immersed in a magnetodielectric medium [48]. Here we extend this quantization scheme to arbitrary multilayer amplifying media.

The structure of the paper is as follows: In Sec. II, we propose a Lagrangian for the electromagnetic field in an amplifying magnetodielectric medium and derive the generating function, which is used in Sec. III for the path-integral quantization. Causal electric and magnetic susceptibilities of the amplifying medium are obtained, both in the frequency interval(s) with gain and in the remaining lossy regions. We calculate the Green tensor and Casimir forces for a multilayer amplifying magnetodielectric medium in Sec. IV, and corresponding numerical results are presented in Sec. V. In Sec. VI, we derive from our Lagrangian a canonical theory that supports the known phenomenological approach to quantization in amplifying magnetodielectrics. We conclude in Sec. VII. Further details of our calculations are given in two appendixes.

\section{FIELD QUANTIZATION}

The quantum electrodynamics of a linearly damped magnetodielectric medium can be described by modeling the medium as two independent reservoirs that interact with the electromagnetic field. Each reservoir contains a continuum of three-dimensional harmonic oscillators that describe the polarizability and magnetizability of the medium [49-55]. We assume the medium is linearly amplifying, at least in one or more finite frequency windows where $\varepsilon_{\mathrm{I}}(\omega)<0$ and/or $\mu_{\mathrm{I}}(\omega)<0$. Despite the striking practical differences between amplifying and lossy media, their theoretical descriptions turn out to be quite similar. We adopt Glauber's inverted oscillator to model the quantum amplifier [34] and use continua of inverted oscillators to describe gain instead of loss in the polarizability and magnetizability of the medium.

We introduce our model for optical media with both gain and loss by first specifying its Lagrangian density in real space,

$$
\mathcal{L}=\mathcal{L}_{\text {EM }}+\mathcal{L}_{\mathrm{e}}+\mathcal{L}_{\mathrm{m}}+\mathcal{L}_{\text {int }},
$$

where the electromagnetic part $\mathcal{L}_{\mathrm{EM}}$ has the standard form $\mathcal{L}_{\mathrm{EM}}=\frac{1}{2} \varepsilon_{0} \mathbf{E}^{2}(\mathbf{x}, t)-\frac{1}{2 \mu_{0}} \mathbf{B}^{2}(\mathbf{x}, t)$. There is gauge freedom to write the electric field $\mathbf{E}=-\partial \mathbf{A} / \partial t-\nabla \phi$ and the magnetic field $\mathbf{B}=\nabla \times \mathbf{A}$ in terms of the scalar and vector potentials $\phi$ and $\mathbf{A}$. For convenience, we choose the Weyl gauge in which the scalar potential vanishes, which allows us to write $\mathbf{E}$ and $\mathbf{B}$ in terms of only the vector potential. The amplifying magnetodielectric medium is modeled with frequency continua of independent vector fields $\mathbf{X}_{\omega}(\mathbf{x}, t)$ and $\mathbf{Y}_{\omega}(\mathbf{x}, t)$, in terms of which we describe the linear electric and magnetic polarization of the medium. Therefore, the material part of the Lagrangian density describing the amplifying medium can now be written as

$$
\begin{gathered}
\mathcal{L}_{\mathrm{e}}=\int_{0}^{\infty} \mathrm{d} \omega\left[\frac{1}{2} \dot{\mathbf{X}}_{\omega}^{2}(\mathbf{x}, t)-\frac{1}{2} \omega^{2} \mathbf{X}_{\omega}^{2}(\mathbf{x}, t)\right] \operatorname{sgn}\left[\varepsilon_{\mathrm{I}}(\omega)\right], \\
\mathcal{L}_{\mathrm{m}}=\int_{0}^{\infty} \mathrm{d} \omega\left[\frac{1}{2} \dot{\mathbf{Y}}_{\omega}^{2}(\mathbf{x}, t)-\frac{1}{2} \omega^{2} \mathbf{Y}_{\omega}^{2}(\mathbf{x}, t)\right] \operatorname{sgn}\left[\mu_{\mathrm{I}}(\omega)\right] .
\end{gathered}
$$

In these Lagrangian densities, for frequencies with positive signs the medium is lossy, and otherwise it is amplifying as modeled with inverted oscillators due to the minus sign. As we see in Sec. VI, this modification leads to field operators that satisfy Maxwell's equations and whose positive-frequency components are associated with both annihilation and creation operators in the case of amplifying media, in accordance with the previous works [37-39].

We define the polarization and magnetization fields of the medium as

$$
\begin{aligned}
\mathbf{P}(\mathbf{x}, t) & =\int_{0}^{\infty} \mathrm{d} \omega f(\mathbf{x}, \omega) \mathbf{X}_{\omega}(\mathbf{x}, t), \\
\mathbf{M}(\mathbf{x}, t) & =\int_{0}^{\infty} \mathrm{d} \omega g(\mathbf{x}, \omega) \mathbf{Y}_{\omega}(\mathbf{x}, t),
\end{aligned}
$$

and assume a linear coupling of the electromagnetic field with these fields,

$$
\begin{aligned}
\mathcal{L}_{\text {int }}(\mathbf{A}, \mathbf{P}, \mathbf{M})= & \mathbf{A}(\mathbf{x}, t) \cdot \dot{\mathbf{P}}(\mathbf{x}, t) \\
& +\nabla \times \mathbf{A}(\mathbf{x}, t) \cdot \mathbf{M}(\mathbf{x}, t) .
\end{aligned}
$$


The $f(\mathbf{x}, \omega)$ and $g(\mathbf{x}, \omega)$ in Eq. (3) are the real-valued scalar coupling functions of the inhomogeneous medium and the electromagnetic field. We have implicity assumed that the medium is isotropic by taking scalar coupling functions. Anisotropy could be included by making them tensors, but this is not pursued here.

With the Lagrangian in a suitable form, we can now define a generating function for our path-integral quantization. For a field theory with only a single scalar canonical field $\varphi$, the generating functional (or partition function) has the form [56]

$$
Z[J]=\int \mathcal{D}[\varphi] \exp \left\{\frac{i}{\hbar} \int \mathrm{d}^{4} x\{\mathcal{L}[\varphi(x)]+J(x) \varphi(x)\}\right\},
$$

where $x \in \mathbb{R}^{4}$ is a space-time coordinate and $J$ is the auxiliary source field associated with the scalar field $\varphi$. In our case, we have several interacting canonical fields in our total Lagrangian (1), so we need to generalize Eq. (5). We first calculate the partition function $Z_{0}$ for the free fields, neglecting their interactions:

$$
\begin{aligned}
& Z_{0}\left[\mathbf{J}_{\mathrm{EM}}, \mathbf{J}_{\mathrm{e}, \omega}, \mathbf{J}_{\mathrm{m}, \omega}\right] \\
& =\int \mathcal{D}[\mathbf{A}] \mathcal{D}\left[\mathbf{X}_{\omega}\right] \mathcal{D}\left[\mathbf{Y}_{\omega}\right] \exp \left\{\frac { i } { \hbar } \int \mathrm { d } ^ { 4 } x \left[\mathcal{L}_{\mathrm{EM}}+\mathcal{L}_{\mathrm{e}}\right.\right. \\
& \left.\left.\quad+\mathcal{L}_{\mathrm{m}}+\mathbf{J}_{0} \cdot \mathbf{A}+\int \mathrm{d} \omega \mathbf{J}_{\mathrm{e}, \omega} \cdot \mathbf{X}_{\omega}+\mathbf{J}_{\mathrm{m}, \omega} \cdot \mathbf{Y}_{\omega}\right]\right\},
\end{aligned}
$$

in terms of the auxiliary source vector field $\mathbf{J}_{\mathrm{EM}}$ for the electromagnetic field and the frequency continua of source fields $\mathbf{J}_{\mathrm{e}, \omega}$ and $\mathbf{J}_{\mathrm{m}, \omega}$ associated with the electric and magnetic polarization fields, respectively. We can write $Z_{0}$ in a more convenient form by employing the four-dimensional version of Gauss's theorem for the vector potential, thereby replacing $\mathcal{L}_{\text {EM }}$ in Eq. (6) by $\left[-\mu_{0}^{-1} \mathbf{A} \cdot(\nabla \times \nabla \times \mathbf{A})-\varepsilon_{0} \mathbf{A} \cdot \partial_{t}^{2} \mathbf{A}\right]$ and by using integration by parts for the polarization fields $\mathbf{X}_{\omega}$ and $\mathbf{Y}_{\omega}$. This gives

$$
\begin{aligned}
Z_{0}\left[\mathbf{J}_{\mathrm{EM}}, \mathbf{J}_{\mathrm{e}, \omega}, \mathbf{J}_{\mathrm{m}, \omega}\right] & \\
= & \int \mathcal{D}[\mathbf{A}] \mathcal{D}\left[\mathbf{X}_{\omega}\right] \mathcal{D}\left[\mathbf{Y}_{\omega}\right] \times \exp \left\{-\frac{i}{\hbar} \int \mathrm{d}^{4} x\right. \\
& \times\left[\frac{1}{2} \mathbf{A} \cdot\left(\mu_{0}{ }^{-1} \nabla \times \nabla \times+\varepsilon_{0} \partial_{t}^{2}\right) \mathbf{A}-\mathbf{J}_{\mathrm{EM}}(x) \cdot \mathbf{A}(x)\right. \\
& -\int \mathrm{d} \omega\left(\mathbf{J}_{\mathrm{e}, \omega}(x) \cdot \mathbf{X}_{\omega}(x)+\mathbf{J}_{\mathrm{m}, \omega}(x) \cdot \mathbf{Y}_{\omega}(x)\right. \\
& +\frac{1}{2} \mathbf{X}_{\omega}(x) \cdot\left(\partial_{t}^{2}-\omega^{2}\right) \mathbf{X}_{\omega}(x) \operatorname{sgn}\left[\varepsilon_{\mathrm{I}}(\omega)\right] \\
& \left.\left.\left.+\frac{1}{2} \mathbf{Y}_{\omega}(x) \cdot\left(\partial_{t}^{2}-\omega^{2}\right) \mathbf{Y}_{\omega}(x) \operatorname{sgn}\left[\mu_{\mathrm{I}}(\omega)\right]\right)\right]\right\} .
\end{aligned}
$$

This partition function is Gaussian since the integrand is quadratic in terms of the fields. Therefore, the functional integration can be performed exactly, and the result is $Z_{0}\left[\mathbf{J}_{\mathrm{EM}}, \mathbf{J}_{\mathrm{e}, \omega}, \mathbf{J}_{\mathrm{m}, \omega}\right]=\exp \left\{i \mathcal{L}_{0} / 2 \hbar\right\}$, in terms of the Lagrangian for noninteracting fields

$$
\begin{aligned}
\mathcal{L}_{0}= & \int \mathrm{d}^{4} x \int \mathrm{d}^{4} x^{\prime} \mathbf{J}_{\mathrm{EM}}(x) \cdot \mathbf{G}_{\mathrm{EM}}^{(0)}\left(x-x^{\prime}\right) \cdot \mathbf{J}_{\mathrm{EM}}\left(x^{\prime}\right) \\
& +\int \mathrm{d}^{3} \mathbf{x} \int \mathrm{d} t \int \mathrm{d} t^{\prime} \int \mathrm{d} \omega
\end{aligned}
$$

$$
\begin{aligned}
& \times\left\{\mathbf{J}_{\mathrm{e}, \omega}(\mathbf{x}, t) \cdot \mathbf{G}_{\mathrm{e}, \omega}\left(t-t^{\prime}\right) \cdot \mathbf{J}_{\mathrm{e}, \omega}\left(\mathbf{x}, t^{\prime}\right) \operatorname{sgn}\left[\varepsilon_{\mathrm{I}}(\omega)\right]\right. \\
& \left.+\mathbf{J}_{\mathrm{m}, \omega}(\mathbf{x}, t) \cdot \mathbf{G}_{\mathrm{m}, \omega}\left(t-t^{\prime}\right) \cdot \mathbf{J}_{\mathrm{m}, \omega}\left(\mathbf{x}, t^{\prime}\right) \operatorname{sgn}\left[\mu_{\mathrm{I}}(\omega)\right]\right\} .
\end{aligned}
$$

Here, the space component of the space-time $x \in \mathbb{R}^{4}$ is indicated in bold by $\mathbf{x} \in \mathbb{R}^{3}$ and the time component is indicated by $t \in \mathbb{R}$. The Green tensor $\mathbf{G}_{\mathrm{EM}}^{(0)}\left(x-x^{\prime}\right)$ for the free electromagnetic field satisfies

$$
\left(\nabla \times \nabla \times+\frac{1}{c^{2}} \frac{\partial^{2}}{\partial t^{2}}\right) \mathbf{G}_{\mathrm{EM}}^{(0)}\left(x-x^{\prime}\right)=\mu_{0} \delta^{4}\left(x-x^{\prime}\right) \mathbf{1}_{3},
$$

describing the propagation of light in time and in free space, whereas the Green tensors $\mathbf{G}_{\mathrm{e}, \omega}\left(t-t^{\prime}\right)=\mathbf{G}_{\mathrm{m}, \omega}\left(t-t^{\prime}\right)$ for the noninteracting electric and magnetic polarization fields describe propagation only in time,

$$
\left(\frac{\partial^{2}}{\partial t^{2}}+\omega^{2}\right) \mathbf{G}_{\mathrm{e} / \mathrm{m}, \omega}\left(t-t^{\prime}\right)=\delta\left(t-t^{\prime}\right) \mathbf{1}_{3},
$$

where $\mathbf{1}_{3}$ denotes the spatial unit tensor. This indicates that the only way to transport energy in the interacting system is via the electromagnetic field. For the same reason, the polarization and magnetization fields in the absence of the electromagnetic field do not lead to a Casimir force [48]. The retarded solution of Eq. (9) in Fourier space is $\mathbf{G}_{\mathrm{e} / \mathrm{m}, \omega}\left(\omega^{\prime}\right)=\mathbf{1}_{3} /\left[\omega^{2}-\left(\omega^{\prime}+i 0^{+}\right)^{2}\right]$. It is convenient to define the source fields $\mathbf{J}_{\mathrm{P}, \mathrm{M}}$ for the electric and magnetic polarization fields as linear combinations of the corresponding frequency continua $\mathbf{J}_{\mathrm{e}, \mathrm{m}}$, namely $\mathbf{J}_{\mathrm{P}}(\mathbf{x}) \equiv \int_{0}^{\infty} \mathrm{d} \omega f(\mathbf{x}, \omega) \mathbf{J}_{\mathrm{e}, \omega}(\mathbf{x}, \omega)$ and $\mathbf{J}_{\mathrm{M}}(\mathbf{x}) \equiv \int_{0}^{\infty} \mathrm{d} \omega g(\mathbf{x}, \omega) \mathbf{J}_{\mathrm{m}, \omega}(\mathbf{x}, \omega)$. With these, the generating functional for the interacting fields can be written in terms of the free generating functional as [56]

$$
\begin{aligned}
Z\left[\mathbf{J}_{\mathrm{EM}}, \mathbf{J}_{\mathrm{P}}, \mathbf{J}_{\mathrm{M}}\right]= & Z_{0}^{-1}\left[\mathbf { 0 , 0 , 0 ] } \operatorname { e x p } \left\{\frac{i}{\hbar} \int \mathrm{d}^{4} x \mathcal{L}_{\mathrm{int}}\right.\right. \\
& \left.\times\left(\frac{\hbar}{i} \frac{\delta}{\delta \mathbf{J}_{\mathrm{EM}}(x)}, \frac{\hbar}{i} \frac{\delta}{\delta \mathbf{J}_{\mathrm{P}}(x)}, \frac{\hbar}{i} \frac{\delta}{\delta \mathbf{J}_{\mathrm{M}}(x)}\right)\right\} \\
& \times Z_{0}\left[\mathbf{J}_{\mathrm{EM}}, \mathbf{J}_{\mathrm{P}}, \mathbf{J}_{\mathrm{M}}\right],
\end{aligned}
$$

where $Z_{0}\left[\mathbf{J}_{\mathrm{EM}}, \mathbf{J}_{\mathrm{P}}, \mathbf{J}_{\mathrm{M}}\right]$ is the free-space partition function, $\mathcal{L}_{\text {int }}$ is given in Eq. (4), and $Z_{0}^{-1}[\mathbf{0 , 0 , 0}]$ is the normalization factor. The exponential in this functional is to be understood as a power series in the coupling functions, that is, by perturbation theory. By using the specific form Eq. (4) for the interaction, we obtain

$$
\begin{aligned}
Z\left[\mathbf{J}_{\mathrm{EM}}, \mathbf{J}_{\mathrm{P}}, \mathbf{J}_{\mathrm{M}}\right]= & Z_{0}^{-1}[\mathbf{0 , 0}, \mathbf{0}] \sum_{n=0}^{\infty} \frac{1}{n !}\left\{-i \hbar \int \mathrm{d}^{4} x\right. \\
& \times\left[\frac{\delta}{\delta \mathbf{J}_{\mathrm{EM}}(x)} \cdot \frac{\partial}{\partial t} \frac{\delta}{\delta \mathbf{J}_{\mathrm{P}}(x)}+\nabla\right. \\
& \left.\left.\times \frac{\delta}{\delta \mathbf{J}_{\mathrm{EM}}(x)} \cdot \frac{\delta}{\delta \mathbf{J}_{\mathrm{M}}(x)}\right]\right\}^{n} \\
& \times Z_{0}\left[\mathbf{J}_{\mathrm{EM}}, \mathbf{J}_{\mathrm{P}}, \mathbf{J}_{\mathrm{M}}\right]
\end{aligned}
$$

Hereby we determined as one of our main results the partition function $Z$ for the interacting fields that describe a magnetodielectric medium with both linear gain and loss. 


\section{GREEN TENSORS AND SUSCEPTIBILITIES OF THE AMPLIFYING MEDIUM}

As we see in the following, the Casimir force can be computed in terms of the electromagnetic Green tensor of the medium. In our zero-temperature field theory, the Green tensors (or propagators) are vacuum expectation values of time-ordered products of field operators, which can be computed as functional derivatives of the partition function (see also Ref. [48]),

$$
\mathbf{G}_{\mathrm{EM}}\left(x, x^{\prime}\right)=-\left.i \hbar \frac{\delta^{2} Z\left[\mathbf{J}_{\mathrm{EM}}, \mathbf{J}_{\mathrm{P}}, \mathbf{J}_{\mathrm{M}}\right]}{\delta \mathbf{J}_{\mathrm{EM}}(x) \delta \mathbf{J}_{\mathrm{EM}}\left(x^{\prime}\right)}\right|_{\mathbf{J}_{\mathrm{EM}}=\mathbf{J}_{\mathrm{P}}=\mathbf{J}_{\mathrm{M}}=\mathbf{0}},
$$

in terms of space-time coordinates $x, x^{\prime}$. The medium described by our Lagrangian (1) in general is not translationally invariant, but it is stationary, and consequently $\mathbf{G}_{\mathrm{EM}}\left(x, x^{\prime}\right)=$ $\mathbf{G}_{\mathrm{EM}}\left(\mathbf{x}, \mathbf{x}^{\prime}, t-t^{\prime}\right)$. After evaluating the functional derivatives of Eq. (12), we obtain a Dyson equation for the Green tensor that after time-Fourier transformation becomes

$$
\begin{aligned}
\mathbf{G}_{\mathrm{EM}}\left(\mathbf{x}, \mathbf{x}^{\prime}, \omega\right)= & \mathbf{G}_{\mathrm{EM}}^{(0)}\left(\mathbf{x}-\mathbf{x}^{\prime}, \omega\right) \\
& +\omega^{2} \int \mathrm{d} \mathbf{x}_{1}\left[\mathbf{G}_{\mathrm{EM}}^{(0)}\left(\mathbf{x}-\mathbf{x}_{1}, \omega\right) \cdot\right. \\
& \times \int \mathrm{d} \omega^{\prime}\left\{\operatorname{sgn}\left[\varepsilon_{\mathrm{I}}\left(\omega^{\prime}\right)\right] f^{2}\left(\mathbf{x}_{1}, \omega^{\prime}\right) \mathbf{G}_{\mathrm{e}, \omega^{\prime}}(\omega)\right\} \\
& \left.\times \mathbf{G}_{\mathrm{EM}}\left(\mathbf{x}_{1}, \mathbf{x}^{\prime}, \omega\right)\right] \\
& +\int \mathrm{d} \mathbf{x}_{1}\left[\mathbf{G}_{\mathrm{EM}}^{(0)}\left(\mathbf{x}-\mathbf{x}_{1}, \omega\right) \times \overleftarrow{\nabla}_{1} \cdot\right. \\
& \times \int \mathrm{d} \omega^{\prime}\left\{\operatorname{sgn}\left[\mu_{\mathrm{I}}\left(\omega^{\prime}\right)\right] g^{2}\left(\mathbf{x}_{1}, \omega^{\prime}\right) \mathbf{G}_{\mathrm{m}, \omega^{\prime}}(\omega)\right\} \\
& \left.\times \nabla_{1} \times \mathbf{G}_{\mathrm{EM}}\left(\mathbf{x}_{1}, \mathbf{x}^{\prime}, \omega\right)\right]
\end{aligned}
$$

This long equation for the Green tensor can be brought into a more familiar form by applying the differential operator $\left(\mu_{0}^{-1} \nabla \times \nabla \times-\omega^{2} \varepsilon_{0} \mathbf{1}_{3}\right)$ to both sides, giving

$$
\begin{aligned}
\nabla & \times\left[\mu^{-1}(\mathbf{x}, \omega) \nabla \times \mathbf{G}_{\mathrm{EM}}\left(\mathbf{x}, \mathbf{x}^{\prime}, \omega\right)\right] \\
& -\frac{\omega^{2} \varepsilon(\mathbf{x}, \omega)}{c^{2}} \mathbf{G}_{\mathrm{EM}}\left(\mathbf{x}, \mathbf{x}^{\prime}, \omega\right)=\mu_{0} \delta^{3}\left(\mathbf{x}-\mathbf{x}^{\prime}\right) \mathbf{1}_{3} .
\end{aligned}
$$

Here we defined the electric permittivity $\varepsilon(\mathbf{x}, \omega)=1+$ $\chi_{\mathrm{e}}(\mathbf{x}, \omega)$ and the inverse magnetic permeability $\mu^{-1}(\mathbf{x}, \omega)=$ $1-\chi_{\mathrm{m}}(\mathbf{x}, \omega)$ of the amplifying magnetodielectric via

$$
\begin{aligned}
& \chi_{\mathrm{e}}(\mathbf{x}, \omega)=\frac{1}{\varepsilon_{0}} \int_{0}^{\infty} \mathrm{d} \omega^{\prime} \frac{f^{2}\left(\mathbf{x}, \omega^{\prime}\right) \operatorname{sgn}\left[\varepsilon_{\mathrm{I}}\left(\omega^{\prime}\right)\right]}{\omega^{\prime 2}-\left(\omega+i 0^{+}\right)^{2}}, \\
& \chi_{\mathrm{m}}(\mathbf{x}, \omega)=\mu_{0} \int_{0}^{\infty} \mathrm{d} \omega^{\prime} \frac{g^{2}\left(\mathbf{x}, \omega^{\prime}\right) \operatorname{sgn}\left[\mu_{\mathrm{I}}\left(\omega^{\prime}\right)\right]}{\omega^{\prime 2}-\left(\omega+i 0^{+}\right)^{2}} .
\end{aligned}
$$

With these definitions and the requirement on the coupling functions that $f^{2}\left(\mathbf{x},-\omega^{*}\right)=f^{2}(\mathbf{x}, \omega)$ and $g^{2}\left(\mathbf{x},-\omega^{*}\right)=$ $g^{2}(\mathbf{x}, \omega)$, the $\varepsilon$ and $\mu$ are complex functions of frequency which satisfy Kramers-Kronig relations [57] and have the properties of response functions, that is, $\varepsilon\left(\mathbf{x},-\omega^{*}\right)=\varepsilon^{*}(\mathbf{x}, \omega)$, and analogously for $\mu$. Purely lossy media would have the further properties $\varepsilon_{\mathrm{I}}(\mathbf{x}, \omega)>0$ and $\mu_{\mathrm{I}}(\mathbf{x}, \omega)>0$, but here we have a model that can describe amplification in some frequency interval(s) as well, for which $\varepsilon_{\mathrm{I}}(\mathbf{x}, \omega)<0$ and/or $\mu_{\mathrm{I}}(\mathbf{x}, \omega)<0$. The functions $\chi_{\mathrm{e}, \mathrm{m}}(\mathbf{x}, \omega)$ have no poles in the upper half of the frequency plane and tend to zero as $\omega \rightarrow \infty$, so that in the time domain, the electric and magnetic susceptibilities $\chi_{\mathrm{e}, \mathrm{m}}(\mathbf{x}, t)$ corresponding to Eq. (15) become proportional to the step function $\Theta(t)$. This is as it should be, since with either gain or loss, the response should be causal.

It is important to stress the generality of our model: If we are given definite functions for the electric permittivity $\varepsilon(\mathbf{x}, \omega)$ and magnetic permeability $\mu(\mathbf{x}, \omega)$ of the gain medium, then we can invert the relations (15) to find the corresponding coupling functions $f(\mathbf{x}, \omega)=\sqrt{2 \omega \varepsilon_{0}\left|\varepsilon_{\mathrm{I}}(\mathbf{x}, \omega)\right| / \pi}$ and $g(\mathbf{x}, \omega)=$ $\sqrt{2 \omega\left|\mu_{I}^{-1}(\mathbf{x}, \omega)\right| / \pi \mu_{0}}$, where the modulus signs ensure that the coupling functions are real-valued both for lossy and for amplifying media. A similar general theory, albeit for purely lossy media, can be found in Refs. [54,55]. Specific choices for the optical functions $\varepsilon$ and $\mu$ will be made for our numerical investigations in Sec. V.

We make some further consistency checks on the pathintegral quantization for amplifying dielectrics. Similar checks for lossy media were performed in Ref. [48]. Recall that the defining equation (14) for the electromagnetic Green tensor is found by functional differentiation of the partition function. This equation enables the identification of the dielectric functions $\varepsilon(\omega)$ and $\mu(\omega)$ for the amplifying medium. Analogously, we can find Green tensors for the material fields in our theory, as well as correlations functions of mixed type. An example of the latter type is

$$
\begin{aligned}
\mathbf{G}_{\mathrm{EM}, \mathrm{P}}\left(\mathbf{x}, \mathbf{x}^{\prime}, \omega\right) & =-\left.i \hbar \frac{\delta^{2} Z\left[\mathbf{J}_{\mathrm{EM}}, \mathbf{J}_{\mathrm{P}}, \mathbf{J}_{\mathrm{M}}\right]}{\delta \mathbf{J}_{\mathrm{EM}}(x) \delta \mathbf{J}_{\mathrm{P}}\left(x^{\prime}\right)}\right|_{\mathbf{J}_{\mathrm{EM}}, \mathbf{J}_{\mathrm{P}}, \mathbf{J}_{\mathrm{M}}=\mathbf{0}} \\
& =i \omega \varepsilon_{0}[\varepsilon(\mathbf{x}, \omega)-1] \mathbf{G}_{\mathrm{EM}}\left(\mathbf{x}, \mathbf{x}^{\prime}, \omega\right) .
\end{aligned}
$$

Analogously we find $\mathbf{G}_{\mathrm{EM}, \mathrm{M}}\left(\mathbf{x}, \mathbf{x}^{\prime}, \omega\right)=\mu_{0}^{-1}\left[1-\mu^{-1}(\mathbf{x}, \omega)\right]$ $\nabla \times \mathbf{G}_{\mathrm{EM}}\left(\mathbf{x}, \mathbf{x}^{\prime}, \omega\right)$, with $\varepsilon(\omega)$ and $\mu(\omega)$ as previously defined in Eqs. (15). This shows that the definition of these response functions for amplifying media can be made uniquely and consistently in the path-integral quantization method. In Sec. VI, we derive the equivalent canonical quantization theory for amplifying dielectrics, with the same Lagrangian (1) as a starting point.

\section{CASIMIR FORCE FOR AMPLIFYING MULTILAYER MEDIA}

\section{A. Derivation of $\boldsymbol{F}_{\mathrm{C}}$ in path-integral formalism}

Here we calculate the Casimir force for two parallel perfectly conducting plates that are separated by a multilayer linearly amplifying medium of total width $d$. Of course, perfect conductors do not exist, and the assumption of linear amplification in reality will break down in an amplifying medium without round-trip losses. Still, an important advantage of our model is that we consider causal optical response functions $\varepsilon(\omega)$ and $\mu(\omega)$, satisfying the Kramers-Kronig relations, as it should for any medium that respects causality, amplifying or not. 
For the single homogeneous amplifying medium, the Casimir force between two plates can be computed as the spatial derivative of the effective action

$$
F_{\mathrm{C}}=\frac{\partial}{\partial d} S_{\mathrm{eff}}(d),
$$

where the effective action is proportional to the logarithm of the partition function,

$$
S_{\text {eff }}(d)=\hbar \ln Z[d] .
$$

Now since for planar structures there are independent TE and TM solutions of Maxwell's equations, the total partition function is the product of $Z_{\mathrm{TE}}[d]$ and $Z_{\mathrm{TM}}[d]$ partition functions, so that the effective action intuitively becomes the sum of TE and TM contributions

$$
S_{\text {eff }}[d]=\hbar\left(\ln Z_{\mathrm{TM}}[d]+\ln Z_{\mathrm{TE}}[d]\right),
$$

and likewise for the Casimir force. The details of the calculation of the partition functions are left to Appendix A, and the calculation yields

$$
Z_{\mathrm{TE}, \mathrm{TM}}=\frac{1}{\sqrt{\operatorname{det} \Gamma_{\mathrm{TE}, \mathrm{TM}}\left(x, y, z_{1}, z_{2}\right)}},
$$

where

$$
\begin{aligned}
& \Gamma_{\mathrm{TM}}\left(x, y, z_{1}, z_{2}\right) \\
& \quad=\left[\begin{array}{ll}
\mathcal{G}_{\mathrm{TM}}\left(x-y, z_{1}, z_{1}\right) & \mathcal{G}_{\mathrm{TM}}\left(x-y, z_{2}, z_{1}\right) \\
\mathcal{G}_{\mathrm{TM}}\left(x-y, z_{1}, z_{2}\right) & \mathcal{G}_{\mathrm{TM}}\left(x-y, z_{2}, z_{2}\right)
\end{array}\right], \\
& \Gamma_{\mathrm{TE}}\left(x, y, z_{1}, z_{2}\right) \\
& \quad=\left[\begin{array}{ll}
-\partial_{z}^{2} \mathcal{G}_{\mathrm{TE}}\left(x-y, z_{1}, z_{1}\right) & -\partial_{z}^{2} \mathcal{G}_{\mathrm{TE}}\left(x-y, z_{2}, z_{1}\right) \\
-\partial_{z}^{2} \mathcal{G}_{\mathrm{TE}}\left(x-y, z_{1}, z_{2}\right) & -\partial_{z}^{2} \mathcal{G}_{\mathrm{TE}}\left(x-y, z_{2}, z_{2}\right)
\end{array}\right] .
\end{aligned}
$$

We see from Eqs. (17)-(21) how the Casimir force is expressed in terms of Green tensors $\mathcal{G}_{\mathrm{TE}, \mathrm{TM}}$, which can be obtained from the Green tensor Eq. (14) by applying a Wick rotation. The explicit form of the Green tensor for planar multilayer dielectric structures was obtained in Ref. [58]. The details of the calculation of the Green tensors for the more general situation of amplifying magnetodielectric multilayer media are summarized in Appendix B.

Before considering multilayer media in more detail, we focus on Casimir force in the presence of a single homogeneous amplifying layer. This is the geometry originally studied by Casimir but now with the vacuum between the conductors replaced by the amplifying medium. For this simple geometry, the Dirichlet and Neumann boundary conditions are formally the same and lead to the same result, so that TE and TM waves each account for half of the Casimir force.

For this case, the Green function (14) in two-dimensional (2D) Fourier space and after Wick rotation can be written as $\mathcal{G}_{\mathrm{EM}}\left(\mathbf{q}, i \omega, z, z^{\prime}\right)=\mu_{0} \mu(i \omega) e^{-\mathcal{Q}\left|z-z^{\prime}\right|} /(2 \mathcal{Q})$, where $\mathcal{Q}(\mathbf{q}, i \omega)=\sqrt{q^{2}+\omega^{2} \varepsilon(i \omega) \mu(i \omega) / c^{2}}$ (see Appendix B).

There is no ambiguity how this square root is to be taken, since $\varepsilon(i \omega)$ and $\mu(i \omega)$ are both real-valued functions of (real) $\omega$. Since both $\varepsilon(\omega)$ and $\mu(\omega)$ have no odd-order zeros in the upper half of the frequency plane and tend to unity in the limit of $|\omega|$ going to infinity, it follows that both $\varepsilon$ and $\mu$ assume positive real values on the positive imaginary frequency axis
[59]. Since consequently $\varepsilon(\omega) \mu(\omega)$ does not have any poles or odd-order zeros in the upper half of the frequency plane, $n(\omega)$ for $\operatorname{Im}(\omega)>0$ is defined as the analytic branch of $\sqrt{\varepsilon(\omega) \mu(\omega)}$ that tends to +1 as $|\omega|$ goes to infinity. (Otherwise, $\sqrt{\varepsilon(\omega) \mu(\omega)}$ would not be an analytic function there and corresponds to materials with so-called absolute instabilities $[60,61]$.) So we find that both $n(\omega)$ and the Green tensor $\mathcal{G}_{\mathrm{EM}}\left(\mathbf{q}, \omega, z, z^{\prime}\right)$ are analytic in the whole upper complex-frequency plane, as it should [62]. By substituting the expression for the Green function $\mathcal{G}_{\mathrm{EM}}\left(\mathbf{q}, i \omega, z, z^{\prime}\right)$ into Eq. (18), the Casimir force per unit area for a homogeneous amplifying medium becomes

$$
\begin{aligned}
F_{\mathrm{C}} & =-\hbar \int \frac{\mathrm{d} \omega \mathrm{d}^{2} \mathbf{q}}{(2 \pi)^{3}} \frac{2 \mathcal{Q}(\mathbf{q}, i \omega)}{e^{2 \mathcal{Q}(\mathbf{q}, i \omega) d}-1} \\
& =-\frac{\hbar}{3 c^{3} \pi^{2}} \int_{0}^{\infty} \mathrm{d} \omega \frac{\omega \frac{\mathrm{d}}{\mathrm{d} \omega}[n(i \omega) \omega]^{3}}{e^{2 n(i \omega) \omega d / c}-1},
\end{aligned}
$$

The last identity in (22) follows from a partial integration over $\omega$ and calculating the $\omega$ derivative of the integral over $q$ [63]. For the empty cavity, the integrals in Eq. (22) can be evaluated exactly, giving the well-known result $F_{\mathrm{C}}=-\hbar c \pi^{2} /\left(240 d^{4}\right)$.

As one of our main results, we find that the Casimir force in the presence of an amplifying medium (22) has the same form as for a purely attenuating medium [48], provided that $\varepsilon(\omega) \mu(\omega)$ for amplifying medium does not have any poles or odd-order zeros in the upper half of the plane. Thus, the actual value of the force for lossy and amplifying media can only follow from the different forms of $\varepsilon(\omega)$ and $\mu(\omega)$ in both cases. The key difference was already stated in the introduction, namely that amplifying media have one or more frequency intervals with $\varepsilon_{\mathrm{I}}(\omega)<0$ or $\mu_{\mathrm{I}}(\omega)<0$ or both, for real frequencies $\omega>0$, whereas lossy media always have $\varepsilon_{\mathrm{I}}(\omega), \mu_{\mathrm{I}}(\omega) \geqslant 0$ for positive real frequencies.

\section{B. Analytical results: Bounds on the Casimir force}

Realistic amplifying media are amplifying in one or more frequency intervals and lossy elsewhere. Since the Casimir force (22) is obtained as an integral over all frequencies, it may well be that the lossy part dominates the total Casimir force. We study these issues numerically in Sec. V B. To understand the effect of amplification on the Casimir force, we first make the further assumption that the medium is fully amplifying, by which we mean that $\varepsilon_{\mathrm{I}}(\omega), \mu_{\mathrm{I}}(\omega) \leqslant 0$ not only for some but for all positive frequencies. We make this admittedly unrealistic assumption to single out the effect of linear amplification on the Casimir force. This gives us some insight, and after that in Sec. V we relax the assumption of full amplification.

Our aim here is to give a bound for the Casimir force in the presence of a fully amplifying medium, similar to the bounds obtained in Ref. [64] for passive dielectric (i.e., nonmagnetic) mirrors, where from causality considerations it follows that the Casimir force on dielectric slabs is always attractive but less so than between two ideal mirrors separated by vacuum.

The force (22) depends on the refractive index $n(i \omega)=$ $\sqrt{\varepsilon(i \omega) \mu(i \omega)}$, in terms of the susceptibilities $\varepsilon$ and $\mu$ that both tend to unity for high frequencies. Causality implies the identity [59]

$$
\varepsilon(i \omega)-1=\frac{2}{\pi} \int_{0}^{\infty} \mathrm{d} \xi \frac{\xi \varepsilon_{\mathrm{I}}(\xi)}{\omega^{2}+\xi^{2}},
$$


and an analogous identity holds for $\mu(\omega)$. It follows and is known that for passive systems, with $\varepsilon_{\mathrm{I}}(\omega)$ and $\mu_{\mathrm{I}}(\omega)$ always positive on the positive real frequency axis, $\varepsilon(i \omega)$ and $\mu(i \omega)$ decrease monotonically from a finite value $\varepsilon_{\text {static }}, \mu_{\text {static }}>1$ (or $+\infty$ for the electric response of metals) at $\omega=0$ down to unity for $\omega \rightarrow \infty$ [59].

We instead apply the identity (23) to amplifying media, for which it also holds as long as they are described by causal response functions. It follows that for fully amplifying systems, with $\varepsilon_{\mathrm{I}}(\omega)<0$ and $\mu_{\mathrm{I}}(\omega)<0$ on the whole positive real frequency axis, $\varepsilon(i \omega)$ and $\mu(i \omega)$ from a finite value $<1$ at $\omega=0$ increase monotonically toward unity for $\omega \rightarrow \infty$. We can say even more, using the fact that causal response functions $\varepsilon(\omega)$ and $\mu(\omega)$ have no zeros in the upper half of the frequency plane [59]: For fully amplifying media, we find that $\varepsilon(i \omega)$ and $\mu(i \omega)$ increase monotonically from finite values $0 \leqslant \varepsilon_{\text {static }}, \mu_{\text {static }} \leqslant 1$ toward unity for $\omega \rightarrow \infty$.

How are these results related to the Casimir force? We have just found that for fully amplifying media, $n(i \omega)=$ $\sqrt{\varepsilon(i \omega) \mu(i \omega)}$ increases monotonically, assuming values between $n_{\text {static }} \geqslant 0$ and 1 . Therefore, there is a one-to-one mapping:

$$
\omega \leftrightarrow s \equiv \omega n(i \omega), \quad \omega, s \in[0, \infty)
$$

This allows us to rewrite the Casimir force for fully amplifying media of Eq. (22) in terms of the new variable $s$ as

$$
F_{\mathrm{C}}=-\frac{\hbar}{c^{3} \pi^{2}} \int_{0}^{\infty} \mathrm{d} s \frac{s^{2} \omega(s)}{e^{2 s d / c}-1} .
$$

Notice that for free space we have $n(i \omega)=1$ and hence $\omega(s)=$ $s$, which immediately gives the well-known Casimir force for vacuum, $F_{\mathrm{C}}^{\mathrm{vac}}=-\hbar c \pi^{2} /\left(240 d^{4}\right)$. Also for the general case $(25)$ we can say more: Since $0 \leqslant n_{\text {static }} \leqslant n(i \omega) \leqslant 1$ for fully amplifying systems, we can invert the relation (24) and find $s \leqslant \omega(s) \leqslant s / n_{\text {static }}$ for all $s$. Combining this with Eq. (25) immediately gives for all separations $d$ the inequalities

$$
\frac{F_{\mathrm{C}}^{\mathrm{vac}}}{n_{\text {static }}} \leqslant F_{\mathrm{C}}^{\text {full amp }} \leqslant F_{\mathrm{C}}^{\mathrm{vac}}=-\frac{\hbar c \pi^{2}}{240 d^{4}} .
$$

In other words, the Casimir force on two ideal conductors separated by a fully amplifying medium of width $d$ is always more attractive than if the medium were a vacuum but it is not more attractive than by a factor $1 / n_{\text {static }}$. In particular, we find no sign change in the Casimir force (no Casimir repulsion) on ideal conductors separated by a homogeneous fully amplifying magnetodielectric medium. These bounds also hold for fully amplifying media that for some frequencies exhibit negative refraction, as numerical examples in Sec. V illustrate.

The bound (26) holds more generally for magnetodielectric media for which $n(i \omega)$ increases and $0 \leqslant n(i \omega) \leqslant 1$. For example, if $\mu(\omega)$ is purely lossy and $\varepsilon(\omega)$ describes full amplification, then the product of the monotonically decreasing $\mu(i \omega)$ and the monotonically increasing $\varepsilon(i \omega)$ may still be a monotonically increasing function between 0 and 1 .

Similarly, for passive media the causal response functions $\varepsilon(\omega)$ and $\mu(\omega)$ have no zeros in the upper half of the frequency plane, and $\varepsilon(i \omega)$ and $\mu(i \omega)$ decrease monotonically toward unity for $\omega \rightarrow \infty$ [59]. Consequently $s / n_{\text {static }} \leqslant \omega(s) \leqslant s$ for

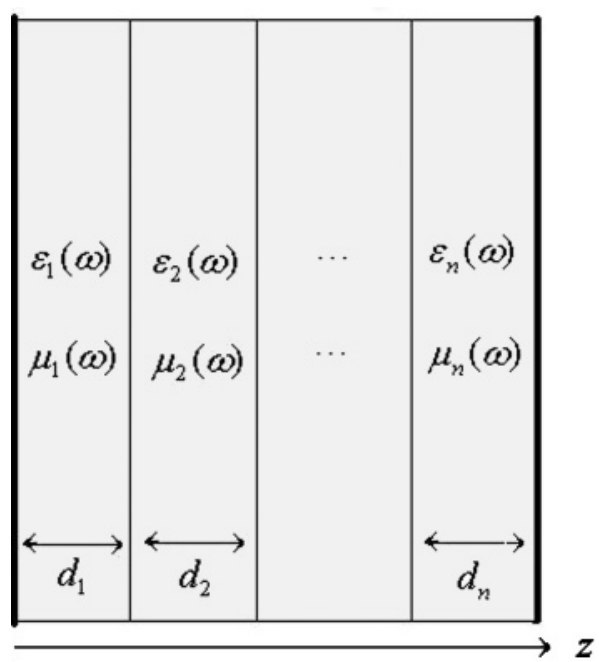

FIG. 1. A planar multilayer magnetodielectric medium sandwiched between two perfect mirrors, with $N$ parallel planar layers labeled by $l=1,2, \ldots, N$. The coordinate system is chosen such that the layers are perpendicular to the $z$ axis. Layer $j$ has thickness $d_{j}$. Each layer is assumed to be homogeneous, isotropic, and of infinite transverse size such that $\varepsilon(\mathbf{x}, \omega)=\varepsilon_{j}(\omega)$ and $\mu(\mathbf{x}, \omega)=\mu_{j}(\omega)$ for $\mathbf{x}$ in layer $j$. The conductors have coordinates $z_{1}$ and $z_{2}$, and their separation $d$ equals $\sum_{j}^{N} d_{j}$.

all $s$, and we find the following inequalities for the Casimir force:

$$
F_{\mathrm{C}}^{\mathrm{vac}} \leqslant F_{\mathrm{C}}^{\text {passive }} \leqslant F_{\mathrm{C}}^{\mathrm{vac}} / n_{\text {static }} \leqslant 0 .
$$

Thus, the Casimir force on two ideal conductors separated by a lossy medium is always attractive, and less attractive than in vacuum, but the force is not reduced by a factor larger than $1 / n_{\text {static }}$. These bounds also hold for passive media that for some frequencies exhibit negative refraction, as numerical examples in Sec. V illustrate.

\section{Casimir forces in amplifying multilayer magnetodielectric media}

Here we generalize our previous results for homogeneous media to planar multilayer geometries, with $N$ parallel planar layers labeled by $l=1,2, \ldots, N$ of thicknesses $d_{l}$, as depicted in Fig. 1. Each layer is assumed to be homogeneous, isotropic, and of infinite transverse size. As is well known for such a planar multilayer geometry, the electromagnetic field can be completely expanded into independent transverse electric (TE) and transverse magnetic (TM) fields that satisfy the same scalar wave equation but differ in their boundary conditions. The Green tensor, that via Eq. (21) determines the Casimir force, can also be separated into TE and TM parts. For multilayer dielectric media, the Green tensor was obtained by Tomaš [58], essentially using a transfer matrix approach, and a generalization to lossy magnetodielectric can be found in Ref. [65]. For our purposes, we need a further generalization, namely the Green tensor for amplifying magnetodielectric multilayer media, and in Appendix B we give a brief derivation and the final result. In general, one finds $N^{2}$ expressions for the Green tensor $\mathcal{G}^{\mathrm{TE}, \mathrm{TM}}\left(\mathbf{q}, i \omega, z, z^{\prime}\right)$, depending on which of the $N$ layers the two coordinates $z$ and $z^{\prime}$ are in, but for the 
Casimir force on the two ideal conductors, Eq. (21) shows that we fortunately only need four of those terms, namely the ones for which $z$ and $z^{\prime}$ both coincide with one of the coordinates $z_{1}$ (boundary of layer 1 ) and $z_{2}$ (boundary of layer $N$ ) of the ideal conductors.

Here we focus on the Casimir force on two ideal conductors separated by three slabs of matter with linear gain and loss, which are spatially homogeneous in the layers 1, 2, and 3 (see Fig. 1). Following the method outlined in Appendix B, we find the four relevant expressions for the Green functions, both for TE and TM polarizations. We give two of them: For $z, z^{\prime}$ both in layer 1 , we find

$$
\begin{aligned}
\mathcal{G}^{\mathrm{TE}, \mathrm{TM}}\left(\mathbf{q}, i \omega, z, z^{\prime}\right)= & \mu_{0} \mu_{1}(i \omega) \mathcal{I}_{11}^{\mathrm{TE}, \mathrm{TM}} \\
& \times \frac{e^{-\mathcal{Q}_{1}\left|z-z^{\prime}\right|}+r_{1+}^{\mathrm{TE}, \mathrm{TM}} e^{-\mathcal{Q}_{1}\left|z+z^{\prime}\right|}}{2 \mathcal{Q}_{1}},
\end{aligned}
$$

while for $z$ in layer 1 and $z^{\prime}$ in layer 3 we obtain

$$
\begin{aligned}
\mathcal{G}^{\mathrm{TE}, \mathrm{TM}}\left(\mathbf{q}, i \omega, z, z^{\prime}\right)= & \mu_{0} \mu_{1}(i \omega) \mathcal{I}_{13}^{\mathrm{TE}, \mathrm{TM}} \\
& \times \frac{t_{1 / 3}^{\mathrm{TE}, \mathrm{TM}} e^{\mathcal{Q}_{1} z} e^{-\mathcal{Q}_{3} z^{\prime}}}{2 \mathcal{Q}_{1}},
\end{aligned}
$$

where $\mathcal{I}_{i j}^{\mathrm{TE}}=1$ and $\mathcal{I}_{i j}^{\mathrm{TM}}=\sqrt{\frac{\varepsilon_{i} \varepsilon_{j}}{\mu_{i} \mu_{j}}}$. Also, the reflection and transmission amplitudes $r_{1+}^{\mathrm{TE}, \mathrm{TM}}$ and $t_{1 / 3}^{\mathrm{TE}, \mathrm{TM}}$ can be calculated with the recursive relations (B6) and (B7). The other two Green functions can be found analogously, for both polarizations. From Eq. (19), we then find the effective action for the amplifying three-layer magnetodielectric medium,

$$
\begin{aligned}
S_{\mathrm{eff}}= & \hbar \int \frac{\mathrm{d} \omega \mathrm{d}^{2} \mathbf{q}}{(2 \pi)^{3}}\left\{\operatorname { l n } \left[\left(1+r_{2,-}^{\mathrm{TM}} e^{-2 \mathcal{Q}_{1} d_{1}}\right)\left(1+r_{2,+}^{\mathrm{TM}} e^{-2 \mathcal{Q}_{3} d_{3}}\right)\right.\right. \\
& \left.-\left(e^{-2 \mathcal{Q}_{1} d_{1}}+r_{2,-}^{\mathrm{TM}}\right)\left(e^{-2 \mathcal{Q}_{3} d_{3}}+r_{2,+}^{\mathrm{TM}}\right) e^{-2 \mathcal{Q}_{2} d_{2}}\right] \\
& +(\mathrm{TM} \rightarrow \mathrm{TE})\}
\end{aligned}
$$

One can check that in the absence of amplification, our expression (30) tends to a known result for lossy media $[2,66]$. In the limit where the two perfect conductors are brought to infinity (i.e., $d_{1}, d_{3} \rightarrow \infty$ ), Eq. (30) reduces to

$$
\begin{aligned}
S_{\text {eff }}= & \hbar \int \frac{\mathrm{d} \omega \mathrm{d}^{2} \mathbf{q}}{(2 \pi)^{3}}\left\{\ln \left[\left(1-r_{2,+}^{\mathrm{TM}} r_{2,-}^{\mathrm{TM}}\right) e^{-2 \mathcal{Q}_{2} d_{2}}\right]\right. \\
& +(\mathrm{TM} \rightarrow \mathrm{TE})\} .
\end{aligned}
$$

This is the generalized Lifshitz formula for the Casimir energy density for amplifying media, in the specific three-layer geometry where two semi-infinite media with permittivities and permeabilities $\varepsilon_{1}, \mu_{1}$ and $\varepsilon_{3}, \mu_{3}$ are separated by a medium of permittivity $\varepsilon_{2}$ and permeability $\mu_{2}$.

The most realistic special case of Eq. (31) is the one where the two semi-infinite media are lossy rather than amplifying, and gain occurs for some frequencies in the middle layer.

\section{NUMERICAL RESULTS}

\section{A. Numerical results for fully amplifying media}

In our numerical investigations, we first choose homogeneous single-resonance Lorentz-oscillator models [67] both

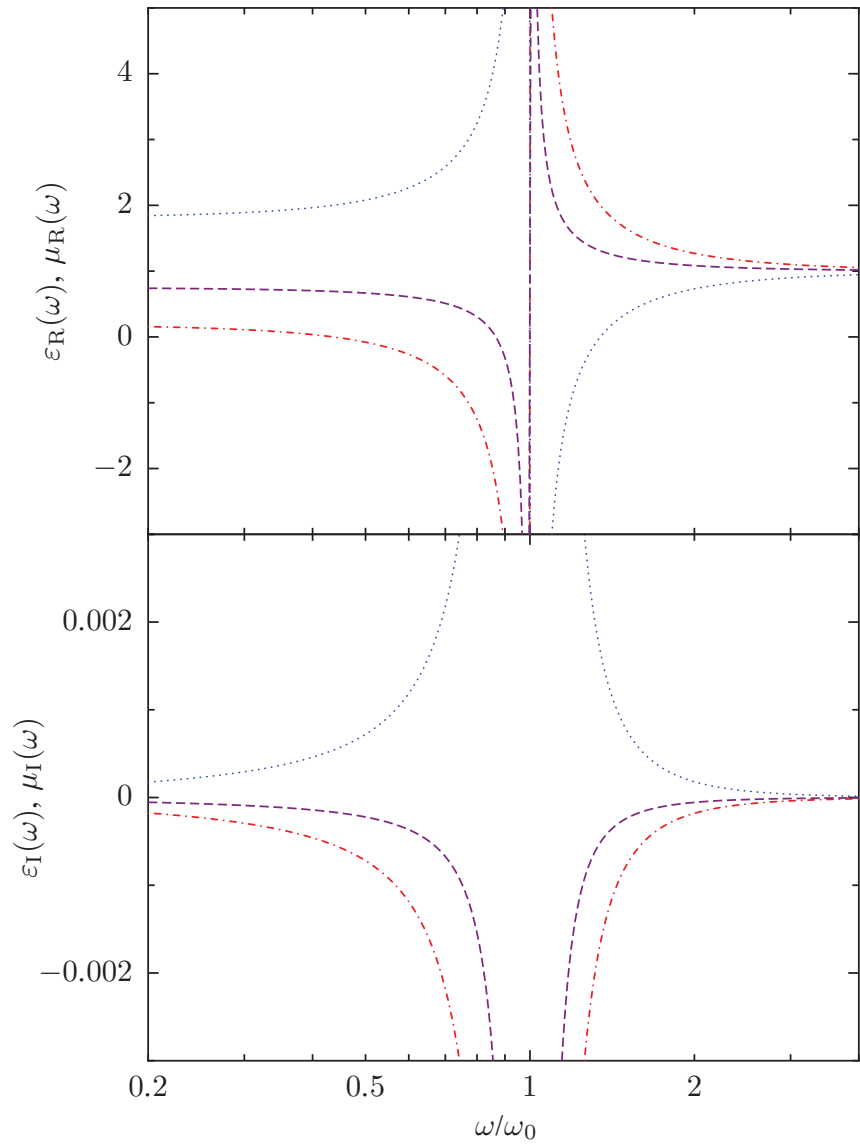

FIG. 2. (Color online) Frequency dispersion of the real (upper panel) and imaginary (lower panel) parts of the electric permittivity and the magnetic permeability, with (real) frequencies on a log scale. The media are assumed to be single-resonance media with resonance frequency $\omega_{0}$ and with $\varepsilon(\omega)=\mu(\omega)$ of the form described by Eq. (32). Parameters for the loss material (dotted curve) are $\omega_{\mathrm{p}(\mathrm{e}, \mathrm{m})} / \omega_{0}=0.9, \gamma_{(\mathrm{e}, \mathrm{m})} / \omega_{0}=0.001$, and for the gain materials the parameters are similar to the loss material but with $\omega_{\mathrm{p}(\mathrm{e}, \mathrm{m})} / \omega_{0}=$ 0.9 (dot-dashed curves), and $\omega_{\mathrm{p}(\mathrm{e}, \mathrm{m})} / \omega_{0}=0.5$ (dashed curves). Note that there are frequency regions where the refractive index becomes negative.

for the electric permittivity $\varepsilon(\omega)$ and for the magnetic permeability $\mu(\omega)$. For simplicity we also assume that the electric and magnetic responses are the same,

$$
\varepsilon(\omega)=\mu(\omega)=1-\frac{\omega_{\mathrm{p}}^{2}}{\omega_{0}^{2}-\omega^{2}-i \gamma \omega},
$$

where $\omega_{\mathrm{p}}$ is the coupling frequency, $\omega_{0}$ is the transverse resonance frequency, and $\gamma$ is the amplification parameter. The minus sign in front of the second term in Eq. (32) accounts for optical gain that arises from population inversion in the medium, and it differs from the usual positive sign for passive systems, consisting, for example, of two-level systems in their ground states. The model (32) is an example of a fully amplifying medium (introduced in Sec. IV B), since $\varepsilon_{\mathrm{I}}(\omega), \mu_{\mathrm{I}}(\omega)<0$ for all positive frequencies, as depicted in Fig. 2.

In Fig. 2, the upper panel depicts the real parts $\varepsilon_{R}(\omega), \mu_{R}(\omega)$. The important thing to notice is that for frequencies $\left(\omega_{0}^{2}-\right.$ 


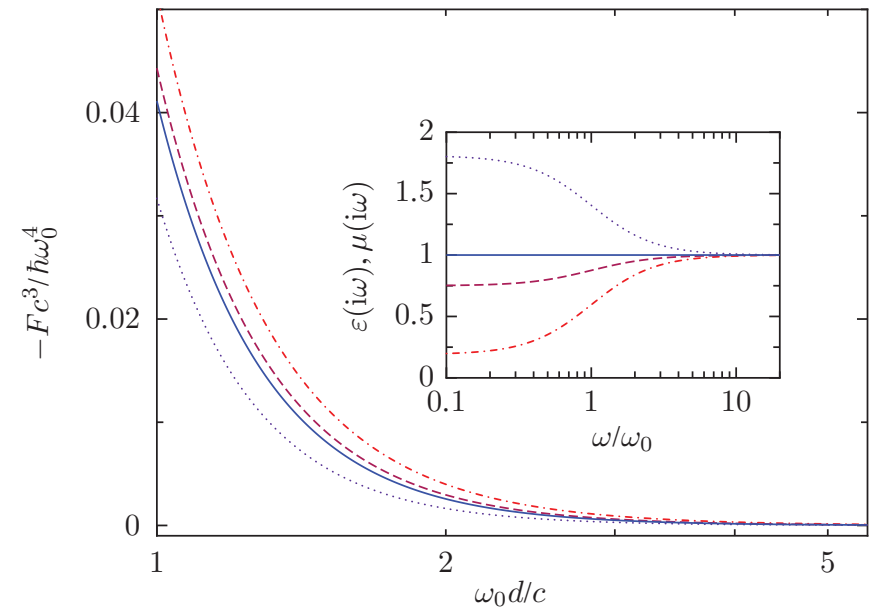

FIG. 3. (Color online) Casimir force $F_{\mathrm{C}}$ per unit area on two perfectly conducting plates, as a function of their separation, for different fully amplifying and lossy dispersive materials between the plates. The parameters are identical to those used in Fig. 2. The solid curve corresponds to a vacuum. The force is given in units of $\hbar \omega_{0}^{4} / c^{3}$ and the separation is given in units of $c / \omega_{0}$. The inset shows $\varepsilon=\mu$ at imaginary frequencies.

$\left.\omega_{\mathrm{p}}^{2}\right)^{1 / 2} \lesssim \omega<\omega_{0}$ both $\varepsilon_{\mathrm{R}}(\omega)$ and $\mu_{\mathrm{R}}(\omega)$ are negative for amplifying media, whereas for passive media they are negative in the region $\omega_{0} \leqslant \omega \lesssim\left(\omega_{0}^{2}+\omega_{\mathrm{p}}^{2}\right)^{1 / 2}$. Thus, our model with $\varepsilon(\omega)=\mu(\omega)$ describes negative refraction, and in particular the perfect-lens situation $n(\omega)=-1$ occurs, or at least $\operatorname{Re}[n(\omega)]=-1$. The lower panel of Fig. 2 depicts the different signs of $\varepsilon_{\mathrm{I}}(\omega), \mu_{\mathrm{I}}(\omega)$ for lossy and for fully amplifying media. The requirement that $\varepsilon(\omega)$ and $\mu(\omega)$ have no simple zeros in the upper half of the frequency plane does not restrict any parameters of Lorentz-oscillator models describing loss, but for the fully amplifying model (32) we must require $\omega_{\mathrm{p}}<\omega_{0}$ [61]. This requirement is usually met, since for natural materials the permeability of the medium typically equals unity and $\gamma_{\mathrm{e}} \ll \omega_{\text {pe }} \ll \omega_{0 \mathrm{e}}[57]$.

In Figure 3, we compare the Casimir force on two perfect planar conductors as described by Eq. (22) for lossy and for fully amplifying homogeneous media, as well as for a vacuum, all as functions of plate separation $d$. The inset 3 shows the real-valued $\varepsilon(i \omega)$, which is indeed monotonically decreasing as discussed in Sec. IV B, here from $\varepsilon_{\text {static }}=1+\omega_{\mathrm{p}}^{2} / \omega_{0}^{2}$ down to unity for lossy media. For amplifying media, $\varepsilon(i \omega)$ is indeed monotonically increasing, from $\varepsilon_{\text {static }}=1-\omega_{\mathrm{p}}^{2} / \omega_{0}^{2}$ toward unity. For some amplifying negative-index geometries, the Casimir force was found to be repulsive [33], but Fig. 3 illustrates our finding of Sec. IV B for a single homogeneous layer: attractive Casimir forces for all plate separations for all homogeneous fully amplifying Kramers-Kronig media, including the media where for some frequencies there is negative refraction. More specifically, it is easily verified that the bounds of Eq. (26) hold in Fig. 3: the attraction for fully amplifying media is always stronger than for plates separated by a vacuum but weaker than $1 / n_{\text {static }}$ times the free-space value. Likewise, the analogous bounds on $F_{\mathrm{C}}$ in Eq. (27) for passive media are also seen to hold in Fig. 3.

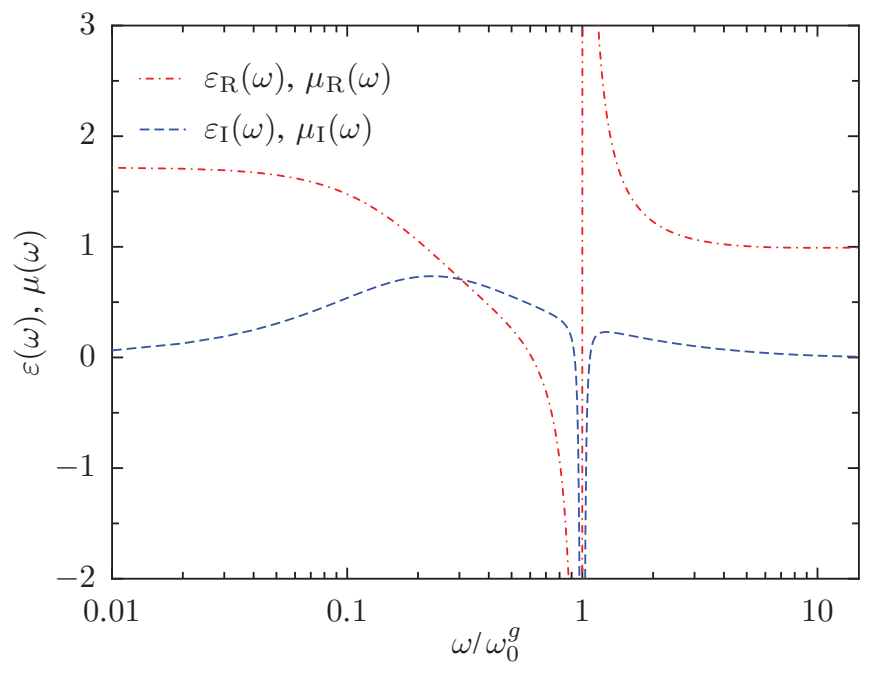

FIG. 4. (Color online) The real and imaginary parts of the electric permittivity and the magnetic permeability in real frequency for the mixed-type gain material with electromagnetic parameters described by Eq. (33): $\omega_{0}^{\mathrm{l}} / \omega_{0}^{\mathrm{g}}=1.5, \omega_{\mathrm{p}}^{\mathrm{g}} / \omega_{0}^{\mathrm{g}}=0.85, \omega_{\mathrm{p}}^{\mathrm{l}} / \omega_{0}^{\mathrm{g}}=1.8, \gamma^{\mathrm{g}} / \omega_{0}^{\mathrm{g}}=$ 0.01 , and $\gamma^{1} / \omega_{0}^{\mathrm{g}}=10$.

\section{B. Numerical results for homogeneous medium with both gain and loss}

The fully amplifying media studied in Secs. IV B and $\mathrm{VA}$ do not occur in nature but give insight in the effect of amplification on Casimir forces. Real amplifying media are typically amplifying in a limited frequency interval and lossy elsewhere. Therefore, we now study the effect of gain in a limited frequency interval on the Casimir force, but we still assume $\varepsilon(\omega)=\mu(\omega)$ for simplicity. We modify the single-resonance model (32) for fully amplifying media by adding a loss term,

$\varepsilon(\omega)=1-\frac{\left(\omega_{\mathrm{p}}^{\mathrm{g}}\right)^{2}}{\left(\omega_{0}^{\mathrm{g}}\right)^{2}-\omega^{2}-i \omega \gamma^{\mathrm{g}}}+\frac{\left(\omega_{\mathrm{p}}^{1}\right)^{2}}{\left(\omega_{0}^{1}\right)^{2}-\omega^{2}-i \omega \gamma^{1}}$.

By our choice of parameters, we describe a medium with gain around $\omega_{0}^{\mathrm{g}}$ and loss elsewhere, as seen in Fig. 4.

In Figure 5, we show the corresponding Casimir force for this medium, as calculated with Eq. (22). More precisely, the figure depicts the difference of the Casimir force with respect to the free-space value, such that a positive value corresponds to a more strongly attractive Casimir force than for free space. For lossy media, we know that this will result in a curve entirely below the horizontal axis, while for fully amplifying media only positive curves would result, as seen in the figure. For the medium (33) with both gain and loss and with $\varepsilon(\omega)=\mu(\omega)$, it follows from the inset of Fig. 5 that $n(i \omega)$ is not monotonically increasing or decreasing but shows more complex behavior: For low frequencies, the curve decreases as for a purely lossy medium. After going through a minimum, the curve increases for a while as fully amplifying media would do monotonically, and then finally it decreases again.

In the main panel of Fig. 5, showing the Casimir force as a function of distance, we see similar behavior: For low frequencies the curve is negative and the force is attractive but weaker than for free space, reminding us of a purely 


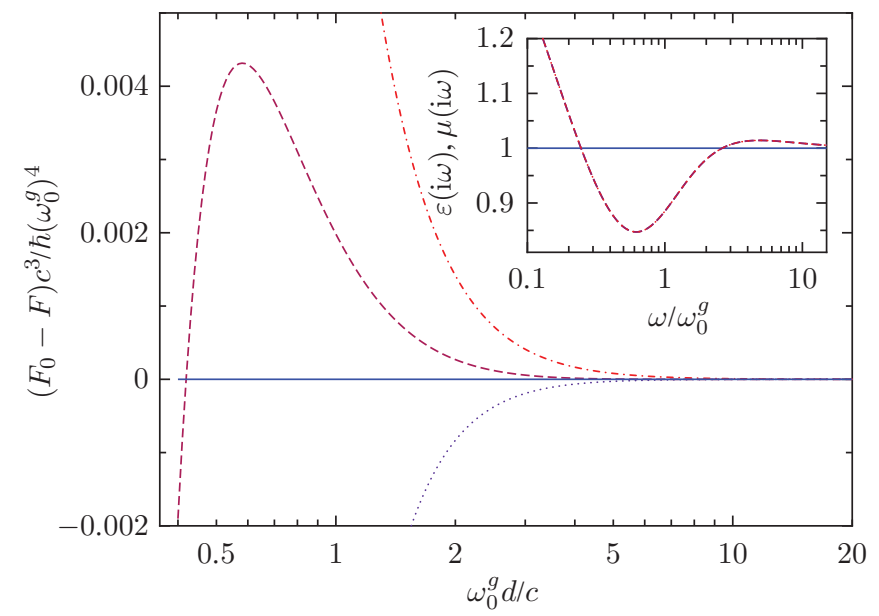

FIG. 5. (Color online) The difference of the Casimir force with respect to the vacuum value, for several dispersive media: lossy, fully amplifying [Eq. (32)], and mixed type [Eq. (33)]. Scaling of the force and of the distance, and parameters for the fully amplifying (dot-dashed) and for lossy media (dotted curves), are similar to those in Fig. 2; parameters for mixed type (dashed curves) are the same as in Fig. 4. The solid curve corresponds to vacuum. The inset shows the corresponding $\omega$ dispersion of $\varepsilon(i \omega)=\mu(i \omega)$ for a mixed-type gain medium.

lossy medium. For intermediate distances, the force is more attractive than for free space, as we have seen for fully amplifying media in Secs. IV B and V A. Finally, for large distances - but this is not clearly visible in the main graph- $-F_{\mathrm{C}}$ again becomes weaker than $F_{\mathrm{C}}^{\mathrm{vac}}$, again reminding us of a lossy medium.

These observations agree with the known fact that at long distances, the main contribution to the Casimir force comes from the low-frequency region, while the force at short distances depends on the high-frequency behavior [26]. This fact follows from the frequency-integral representation (22) of the force. From Fig. 3 for the fully amplifying media this relation was not evident, but in Fig. 5 it is: Gain at a finite frequency interval around $\omega_{0}^{\mathrm{g}}$ may lead to Casimir forces that are more attractive than in free space for a finite interval of plate separations, roughly around a separation $d=\lambda_{0}^{\mathrm{g}} /(2 \pi)$, in terms of the resonance wavelength $\lambda_{0}^{\mathrm{g}}=2 \pi c / \omega_{0}^{\mathrm{g}}$. For closer or more distant separations, the lossy character of the gain-and-loss medium dominates the Casimir force, so that $F_{\mathrm{C}}^{\text {vac }}<F_{\mathrm{C}}<0$. As for the fully amplifying media, we do not find Casimir repulsion for homogeneous media with both gain and loss.

\section{CANONICAL QUANTIZATION OF ELECTROMAGNETIC FIELD IN AMPLIFYING MEDIUM}

In the previous sections, we made a shortcut from the Lagrangian via the partition function and Green tensor to calculate the Casimir force for amplifying media. To find the Casimir force, we did not need to perform explicitly a canonical quantization for amplifying media based on our Lagrangian. The reason to do this here is to make contact with other approaches and to provide an underlying canonical theory for the phenomenological quantum electrodynamics for amplifying media that was developed in recent years [35-39].

The starting point is the Lagrangian (1), with the vector potential $\mathbf{A}$, and the continua of polarization operators $\mathbf{X}_{\omega}$ and $\mathbf{Y}_{\omega}$ as canonical fields with the following canonically conjugate fields:

$$
\begin{gathered}
-\varepsilon_{0} \mathbf{E}(\mathbf{x}, t)=\frac{\delta \mathcal{L}}{\delta \dot{\mathbf{A}}(\mathbf{x}, t)}=\varepsilon_{0} \dot{\mathbf{A}}(\mathbf{x}, t), \\
\mathbf{Q}_{\omega}(\mathbf{x}, t)=\frac{\delta \mathcal{L}}{\delta \dot{\mathbf{X}}_{\omega}(\mathbf{x}, t)} \\
=f(\omega, \mathbf{x}) \mathbf{A}(\mathbf{x}, t)+\operatorname{sgn}\left[\varepsilon_{\mathrm{I}}(\omega)\right] \dot{\mathbf{X}}_{\omega}(\mathbf{x}, t), \\
\Pi_{\omega}(\mathbf{x}, t)=\frac{\delta \mathcal{L}}{\delta \dot{\mathbf{Y}}_{\omega}(\mathbf{x}, t)}=g(\omega, \mathbf{x}) \nabla \times \mathbf{A}(\mathbf{x}, t) \\
+\operatorname{sgn}\left[\mu_{\mathrm{I}}(\omega)\right] \dot{\mathbf{Y}}_{\omega}(\mathbf{x}, t) .
\end{gathered}
$$

Here we find $-\varepsilon_{0} \mathbf{E}$ as the canonical conjugate to the vector potential, as in Refs. [37-39,49-51]. Apart from the subtlety with the sign functions in Eqs. (34) that discriminate between the frequency intervals where there is gain and loss, the canonical quantization of the fields can proceed in a standard fashion by demanding equal-time commutation relations among the variables and their conjugates,

$$
\begin{aligned}
& {\left[A_{i}(\mathbf{x}, t),-\varepsilon_{0} E_{j}\left(\mathbf{x}^{\prime}, t\right)\right]=i \hbar \delta_{i j} \delta^{\perp}\left(\mathbf{x}-\mathbf{x}^{\prime}\right), } \\
& {\left[X_{\omega, i}(\mathbf{x}, t), Q_{\omega^{\prime}, j}\left(\mathbf{x}^{\prime}, t\right)\right]=} i \hbar \delta_{i j} \\
& \times \delta\left(\omega-\omega^{\prime}\right) \delta^{3}\left(\mathbf{x}-\mathbf{x}^{\prime}\right), \\
& {\left[Y_{\omega, i}(\mathbf{x}, t), \Pi_{\omega^{\prime}, j}\left(\mathbf{x}^{\prime}, t\right)\right]=} i \hbar \delta_{i j} \\
& \times \delta\left(\omega-\omega^{\prime}\right) \delta^{3}\left(\mathbf{x}-\mathbf{x}^{\prime}\right),
\end{aligned}
$$

and all other equal-time commutators vanish. Using the Lagrangian (1) and the expression for the canonical conjugate variables in (34), we obtain the Hamiltonian density

$$
\begin{aligned}
\mathcal{H}= & \frac{1}{2} \varepsilon_{0} \mathbf{E}^{2}(\mathbf{x}, t)+\frac{\mathbf{B}^{2}(\mathbf{x}, t)}{2 \mu_{0}} \\
& +\frac{1}{2} \int_{0}^{\infty} \mathrm{d} \omega \operatorname{sgn}\left[\varepsilon_{\mathrm{I}}(\omega)\right]\left\{\dot{\mathbf{X}}_{\omega}^{2}(\mathbf{x}, t)+\omega^{2} \mathbf{X}_{\omega}^{2}(\mathbf{x}, t)\right\} \\
& +\frac{1}{2} \int_{0}^{\infty} \mathrm{d} \omega \operatorname{sgn}\left[\mu_{\mathrm{I}}(\omega)\right]\left\{\dot{\mathbf{Y}}_{\omega}^{2}(\mathbf{x}, t)+\omega^{2} \mathbf{Y}_{\omega}^{2}(\mathbf{x}, t)\right\} .
\end{aligned}
$$

Maxwell's equations can now be obtained from the Heisenberg equations of motion for the vector potential and the transverse electric field and from the commutation relation (35),

$$
\begin{aligned}
& \dot{\mathbf{A}}(\mathbf{x}, t)=-\mathbf{E}(\mathbf{x}, t), \\
& \varepsilon_{0} \dot{\mathbf{E}}(\mathbf{x}, t)=\frac{\nabla \times \nabla \times \mathbf{A}(\mathbf{x}, t)}{\mu_{0}}-\nabla \times \mathbf{M}(\mathbf{x}, t)-\dot{\mathbf{P}}(\mathbf{x}, t) .
\end{aligned}
$$

Using the definitions $\mathbf{D}=\varepsilon_{0} \mathbf{E}+\mathbf{P}$ and $\mathbf{H}=\mathbf{B} / \mu_{0}-\mathbf{M}$ for the displacement field and the magnetic field strength, respectively, Eqs. (37) result in $\dot{\mathbf{D}}(\mathbf{x}, t)=\nabla \times \mathbf{H}(\mathbf{x}, t)$ and $\dot{\mathbf{B}}(\mathbf{x}, t)=$ $-\nabla \times \mathbf{E}(\mathbf{x}, t)$, as expected. In a similar fashion, the Heisenberg equation of motion for the dynamical variables $\mathbf{X}_{\omega}$ and $\mathbf{Y}_{\omega}$ leads to

$$
\begin{aligned}
& \ddot{\mathbf{X}}_{\omega}(\mathbf{x}, t)=-\omega^{2} \mathbf{X}_{\omega}(\mathbf{x}, t)+\operatorname{sgn}\left[\varepsilon_{\mathrm{I}}(\omega)\right] f(\mathbf{x}, \omega) \mathbf{E}(\mathbf{x}, t), \\
& \ddot{\mathbf{Y}}_{\omega}(\mathbf{x}, t)=-\omega^{2} \mathbf{Y}_{\omega}(\mathbf{x}, t)+\operatorname{sgn}\left[\mu_{\mathrm{I}}(\omega)\right] g(\mathbf{x}, \omega) \mathbf{B}(\mathbf{x}, t),
\end{aligned}
$$


with formal solution

$$
\begin{aligned}
\mathbf{X}_{\omega}(\mathbf{x}, t)= & \left(\dot{\mathbf{X}}_{\omega}(\mathbf{x}, 0) \frac{\sin \omega t}{\omega}+\mathbf{X}_{\omega}(\mathbf{x}, 0) \cos \omega t\right) \\
& +f(\mathbf{x}, \omega) \operatorname{sgn}\left[\varepsilon_{\mathrm{I}}(\omega)\right] \\
& \times \int_{0}^{t} \mathrm{~d} t^{\prime} \frac{\sin \omega\left(t-t^{\prime}\right)}{\omega} \mathbf{E}\left(\mathbf{x}, t^{\prime}\right),
\end{aligned}
$$

and likewise for $\mathbf{Y}_{\omega}(\mathbf{x}, t)$. To facilitate the calculations, let us introduce the following annihilation operators

$$
\begin{aligned}
& d_{j}(\mathbf{x}, \omega, t)=\frac{1}{\sqrt{2 \hbar \omega}}\left[\omega \mathrm{X}_{\omega, j}(\mathbf{x}, t)+i \mathrm{Q}_{\omega, j}(\mathbf{x}, t)\right], \\
& b_{j}(\mathbf{x}, \omega, t)=\frac{1}{\sqrt{2 \hbar \omega}}\left[\omega \mathrm{Y}_{\omega, j}(\mathbf{x}, t)+i \Pi_{\omega, j}(\mathbf{x}, t)\right],
\end{aligned}
$$

where $j=1,2,3$ labels three orthogonal spatial directions. Their commutation relations follow immediately from Eq. (35),

$$
\begin{aligned}
& {\left[d_{j}(\mathbf{x}, \omega, t), d_{j^{\prime}}^{\dagger}\left(\mathbf{x}^{\prime}, \omega^{\prime}, t\right)\right]=\delta_{j j^{\prime}} \delta\left(\omega-\omega^{\prime}\right) \delta\left(\mathbf{x}-\mathbf{x}^{\prime}\right),} \\
& {\left[b_{j}(\mathbf{x}, \omega, t), b_{j^{\prime}}^{\dagger}\left(\mathbf{x}^{\prime}, \omega^{\prime}, t\right)\right]=\delta_{j j^{\prime}} \delta\left(\omega-\omega^{\prime}\right) \delta\left(\mathbf{x}-\mathbf{x}^{\prime}\right) .}
\end{aligned}
$$

Now by inverting the relations (40) and substituting the result into Eqs. (3), the polarization and magnetization fields of the magnetodielectric medium can be written in terms of creation and annihilation operators as

$$
\begin{gathered}
\mathbf{P}(\mathbf{x}, t)=\varepsilon_{0} \int_{0}^{\infty} \mathrm{d} t^{\prime} \chi_{\mathrm{e}}\left(\mathbf{x}, t-t^{\prime}\right) \mathbf{E}\left(\mathbf{x}, t^{\prime}\right)+\mathbf{P}^{\mathrm{N}}(\mathbf{x}, t), \\
\mathbf{M}(\mathbf{x}, t)=\frac{1}{\mu_{0}} \int_{0}^{\infty} \mathrm{d} t^{\prime} \chi_{\mathrm{m}}\left(\mathbf{x}, t-t^{\prime}\right) \mathbf{B}\left(\mathbf{x}, t^{\prime}\right)+\mathbf{M}^{\mathrm{N}}(\mathbf{x}, t),
\end{gathered}
$$

with susceptibilities $\chi_{\mathrm{m}, \mathrm{e}}$ as defined in Eq. (15). The fields $\mathbf{P}^{\mathrm{N}}(\mathbf{x}, t)$ and $\mathbf{M}^{\mathrm{N}}(\mathbf{x}, t)$ are the electric and magnetic polarization noise densities associated with absorption and amplification. As in the phenomenological method, we can separate the noise operators into positive- and negative-frequency parts $\mathbf{P}^{\mathrm{N}}=$ $\mathbf{P}^{\mathrm{N}(+)}+\mathbf{P}^{\mathrm{N}(-)}$ with $\mathbf{P}^{\mathrm{N}(-)}=\left[\mathbf{P}^{\mathrm{N}(+)}\right]^{\dagger}$ and analogously for $\mathbf{M}^{\mathrm{N}}$, where

$$
\begin{aligned}
\mathbf{P}^{\mathrm{N}(+)}(\mathbf{x}, t)= & \int_{0}^{\infty} \mathrm{d} \omega \sqrt{\frac{\hbar \varepsilon_{0}\left|\varepsilon_{\mathrm{I}}(\omega)\right|}{\pi}}\left\{d_{i}(\mathbf{x}, \omega, 0) \Theta\left[\varepsilon_{\mathrm{I}}(\omega)\right]\right. \\
& \left.+d_{i}^{\dagger}(\mathbf{x}, \omega, 0) \Theta\left[-\varepsilon_{\mathrm{I}}(\omega)\right]\right\} e^{-i \omega t}, \\
\mathbf{M}^{\mathrm{N}(+)}(\mathbf{x}, t)= & \int_{0}^{\infty} \mathrm{d} \omega \sqrt{\frac{\hbar\left|\mu_{\mathrm{I}}^{-1}(\omega)\right|}{\pi \mu_{0}}}\left\{b_{i}(\mathbf{x}, \omega, 0) \Theta\left[\mu_{\mathrm{I}}(\omega)\right]\right. \\
& \left.+b_{i}^{\dagger}(\mathbf{x}, \omega, 0) \Theta\left[-\mu_{\mathrm{I}}(\omega)\right]\right\} e^{-i \omega t},
\end{aligned}
$$

In fact, these equations are the starting point in Refs. [37-39] to the phenomenological quantization of the electromagnetic field in amplifying magnetodielectric media.

If we now take the time derivative of Eq. (37) and use Eq. (42), this yields the frequency-domain wave equation for the positive-frequency part of the vector potential

$$
\begin{aligned}
\nabla & \times\left[\mu^{-1}(\mathbf{x}, \omega) \nabla \times \mathbf{A}^{(+)}(\mathbf{x}, \omega)\right]-\frac{\omega^{2}}{c^{2}} \varepsilon(\mathbf{x}, \omega) \mathbf{A}^{(+)}(\mathbf{x}, \omega) \\
& =-i \mu_{0} \omega \mathbf{P}^{\mathrm{N}(+)}(\mathbf{x})+\mu_{0} \nabla \times \mathbf{M}^{\mathrm{N}(+)}(\mathbf{x}, \omega)
\end{aligned}
$$

This equation can be solved as

$$
\begin{aligned}
\mathbf{A}^{(+)}(\mathbf{x}, t)= & \frac{1}{2 \pi} \int_{0}^{\infty} \mathrm{d} \omega \int \mathrm{d}^{3} \mathbf{x}^{\prime} \mathbf{G}\left(\mathbf{x}, \mathbf{x}^{\prime}, \omega\right) . \\
& \times\left[-i \omega \mathbf{P}^{\mathrm{N}(+)}\left(\mathbf{x}^{\prime}, \omega\right)\right. \\
& \left.+\nabla \times \mathbf{M}^{\mathrm{N}(+)}\left(\mathbf{x}^{\prime}, \omega\right)\right] e^{-i \omega t}
\end{aligned}
$$

where the Green tensor $\mathbf{G}\left(\mathbf{x}, \mathbf{x}^{\prime}, \omega\right)$ is the solution of Eq. (14).

The equations (43) and (45) and the commutation relations are the same as obtained from the phenomenological method [35-39]. Therefore, with our Lagrangian (1) and the canonical quantization performed here, we formulated a microscopic basis for the phenomenological quantization of the electromagnetic field in amplifying magnetodielectric media.

\section{CONCLUSIONS AND DISCUSSION}

The electromagnetic field in an amplifying magnetodielectric medium was quantized with a path-integral technique. We determined correlation functions of different fields and found electric and magnetic susceptibilities of the amplifying medium that are consistent with causality. We determined the Green functions in amplifying planar multilayer magnetodielectrics and used this to calculate the Casimir energy and force in such media.

The calculations show that the form of the Casimir force as a functional of the dielectric functions $\varepsilon(\mathbf{x}, \omega), \mu(\mathbf{x}, \omega)$ does not change significantly as compared to passive media, but some caution is needed, especially regarding the signs of wavevector components in amplifying negative-index materials in the direction normal to the planes.

Here we studied systems that have both gain and a negative index, and to that end we assumed $\varepsilon(\omega)=\mu(\omega)$ in our numerical calculations, not because this would be simple to realize in experiments but rather to gain insight. The concept was introduced of fully amplifying media, that is, amplifying at all frequencies. The advantage of our approach is that our optical functions $\varepsilon(\omega), \mu(\omega)$ are defined at all frequencies and are causal. For two conductors separated by a homogeneous passive medium, we find that the Casimir force satisfies the bounds of Eq. (27), so that it is always attractive and less so than if the medium were replaced by vacuum. On the other hand, for a homogeneous fully amplifying medium, we find the bounds (26) that the Casimir force is finite and always more attractive than in vacuum.

Both bounds are remarkable insofar that both the fully amplifying and the passive media with $\varepsilon(\omega)=\mu(\omega)$ may have negative refraction in a large frequency interval, whereas for some other planar geometries it was reported that negative refraction may lead to Casimir forces that may become repulsive [33], and also that gain would lead to repulsive Casimir forces [32,33]. This is not a contradiction, however, but rather shows that negative refractive indices or gain do not automatically imply a sign change of the Casimir force and that the sign of the force strongly depends on the geometry also for amplifying and negative-index materials.

The bounds (26) and (27) for homogeneous fully amplifying and passive media have in common that the force is bound by the free-space Casimir force on the one side and by the free-space Casimir force divided by the static refractive index 
$n_{\text {static }}$ on the other. It does not matter whether for microwaves or optical frequencies the medium has a negative refractive index, as long as the static refractive index stays the same. In that sense, the Casimir force has little to do with optics, both for passive and for amplifying media. Reference [26] also stresses the importance of the low-frequency behavior, and here we found an illustrative example also for amplifying systems.

Some observables have divergent values in models of linear amplification, especially for geometries with round-trip gain. Our simple geometry of perfect conductors separated by a single amplifying medium will even exhibit round-trip gain for all frequencies for which there is amplification. Nevertheless, we find that the Casimir force on the conductors is finite, bounded by the inequalities of Eq. (26). This could be explained by the fact that the Casimir force is a vacuum force, so that there are no photons present that are amplified indefinitely.

Casimir forces in amplifying media are only beginning to be explored, and we considered only linear amplification. This is not always a realistic model, especially for geometries where the linear-amplification model predicts round-trip gain. It is an open challenge to calculate Casimir forces in the presence of media with nonlinear amplification, for example, with parametric amplification. A possible route could be to generalize the Lagrangian for passive nonlinear media of Ref. [68] to gain media, and the result would be a generalization of our Lagrangian of Eq. (1) to nonlinearly amplifying media.

Finally, we carried out a canonical quantization of the electromagnetic field in an arbitrary linear amplifying and/or passive medium and showed that the resulting field operators satisfy the macroscopic Maxwell equations for an arbitrary linearly amplifying and/or passive medium. The resulting theory is valid for all linear, inhomogeneous, amplifying, and/or passive magnetodielectric media with dielectric functions that satisfy the Kramers-Kronig relations. The postulates of the phenomenological theory [35-39] that serve as its starting point were here derived by canonical quantization.

\section{ACKNOWLEDGMENTS}

E.A. thanks the Technical University of Denmark for hospitality and the University of Isfahan and the Iranian Ministry of Science, Research, and Technology for financial support. M.W. acknowledges financial support by the Danish Research Council for Technology and Production Sciences (FTP Grant No. 274-07-0080).

\section{APPENDIX A: GENERATING FUNCTION IN PRESENCE OF DIRICHLET AND NEUMANN BOUNDARY CONDITIONS}

Here we calculate the generating function for two perfectly conducting plates surrounding an amplifying planar multilayer system. We are brief, as most of the calculation is identical to the case of lossy media; see Ref. [48], for example.
We can consider TE and TM polarized waves separately. On the two plates (labeled by $\alpha$ ) they satisfy Dirichlet or Neumann boundary conditions, respectively:

$$
\begin{gathered}
\left.\varphi_{\mathrm{TM}}\right|_{S_{\alpha}}=0, \quad \alpha=1,2, \\
\left.\partial_{\mathrm{n}} \varphi_{\mathrm{TE}}\right|_{S_{\alpha}}=0, \quad \alpha=1,2,
\end{gathered}
$$

on each surface $S_{\alpha}$, where $\partial_{\mathrm{n}}$ is the normal derivative of the surface $S_{\alpha}$ pointing into the space between the two plates. To obtain the partition function in four-dimensional (4D) Euclidean space from the Lagrangian (1), we made a Wick rotation so that the signature of space-time changes from Minkowski to Euclidean.

In the 4D Euclidean space, the plates are parameterized by $\mathcal{X}_{1}\left(\mathbf{x}, z_{1}, i t\right)$ and $\mathcal{X}_{2}\left(\mathbf{x}, z_{2}, i t\right)$, where $\mathbf{x}=(x, y)$. The Dirichlet or Neumann boundary conditions corresponding to the constraints (A1) can be imposed by inserting $\delta$ functions which can be expressed in terms of auxiliary fields $\psi_{\alpha}\left(\mathcal{X}_{\alpha}\right)$ as follows $[47,69]$ :

$$
\begin{array}{r}
\delta\left[\varphi\left(\mathcal{X}_{\alpha}\right)\right]=\int \mathcal{D}\left[\psi_{\alpha}\left(\mathcal{X}_{\alpha}\right)\right] e^{i \int \mathrm{d} \mathcal{X}_{\alpha} \psi\left(\mathcal{X}_{\alpha}\right) \varphi\left(\mathcal{X}_{\alpha}\right)}, \\
\delta\left[\partial_{n} \varphi\left(\mathcal{X}_{\alpha}\right)\right]=\int \mathcal{D}\left[\psi_{\alpha}\left(\mathcal{X}_{\alpha}\right)\right] e^{i \int \mathrm{d} \mathcal{X}_{\alpha} \psi\left(\mathcal{X}_{\alpha}\right) \partial_{n} \varphi\left(\mathcal{X}_{\alpha}\right)} .
\end{array}
$$

Using Eqs. (A2), the partition function (5) in Euclidean space can be written as

$$
\begin{gathered}
Z_{\mathrm{TM}}=Z_{0}^{-1} \int \mathcal{D}[\varphi] \prod_{a=1}^{2} \delta\left[\varphi\left(\mathcal{X}_{\alpha}\right)\right] e^{-S_{\mathrm{E}, \mathrm{TM}}[\varphi]}, \\
Z_{\mathrm{TE}}=Z_{0}^{-1} \int \mathcal{D}[\varphi] \prod_{a=1}^{2} \delta\left[\partial_{\mathrm{n}} \varphi\left(\mathcal{X}_{\alpha}\right)\right] e^{-S_{\mathrm{E}, \mathrm{TE}}[\varphi]}
\end{gathered}
$$

where the Euclidean actions $S_{\mathrm{E}, \mathrm{TM} / \mathrm{TE}}(\varphi)$ are defined as

$$
\begin{aligned}
& S_{\mathrm{E}, \mathrm{TM}}[\varphi]=\int \mathrm{d}^{4} x\{\mathcal{L}[\varphi(x)] \\
& \left.+\varphi(x) \sum_{\alpha=1}^{2} \int \mathrm{d} \mathcal{X} \delta\left(\mathcal{X}-\mathcal{X}_{\alpha}\right) \psi_{\alpha}(x)\right\}, \\
& S_{\mathrm{E}, \mathrm{TE}}[\varphi]=\int \mathrm{d}^{4} x\{\mathcal{L}[\varphi(x)] \\
& \left.+\varphi(x) \sum_{\alpha=1}^{2} \int \mathrm{d} \mathcal{X} \delta\left(\mathcal{X}-\mathcal{X}_{\alpha}\right) \partial_{n} \psi_{\alpha}(x)\right\} .
\end{aligned}
$$

By comparing Eqs. (A4) and (11), we can rewrite Eqs. (A3) as

$$
\begin{aligned}
Z_{\mathrm{TM}}= & \int \prod_{\alpha=1}^{2} \mathcal{D}\left[\psi_{\alpha}(x)\right] \\
& \times Z\left(\sum_{\alpha=1}^{2} \int \mathrm{d} \mathcal{X} \delta\left(\mathcal{X}-\mathcal{X}_{\alpha}\right) \psi_{\alpha}(\mathcal{X})\right) \\
Z_{\mathrm{TE}}= & \int \prod_{\alpha=1}^{2} \mathcal{D}\left[\psi_{\alpha}(x)\right] \\
& \times Z\left(\sum_{\alpha=1}^{2} \int \mathrm{d} \mathcal{X} \delta\left(\mathcal{X}-\mathcal{X}_{\alpha}\right) \partial_{n} \psi_{\alpha}(\mathcal{X})\right)
\end{aligned}
$$


where the $Z(\ldots)$ in the two integrands are the generating functionals of interacting fields defined in Eq. (11) with imaginary time. From Eqs. (A5) and (13), the respective partition functions can be written as

$$
Z_{\mathrm{TM} / \mathrm{TE}}=\int \prod_{\alpha=1}^{2} \mathcal{D}\left[\psi_{\alpha}\left(X_{\alpha}\right)\right] e^{-S_{\mathrm{eff}, \mathrm{TM} / \mathrm{TE}}\left(\psi_{\alpha}\right)},
$$

where the effective actions $S_{\mathrm{eff}, \mathrm{TM}}$ and $S_{\mathrm{eff}, \mathrm{TE}}$ are given by

$$
\begin{aligned}
& S_{\text {eff,TM }}\left(\psi_{1}, \psi_{2}\right) \\
& =\frac{1}{2} \sum_{\alpha, \beta} \iint d \mathcal{X}_{\alpha} d \mathcal{X}_{\beta} \psi_{\alpha}\left(\mathcal{X}_{\alpha}\right) \mathcal{G}\left(\mathcal{X}_{\alpha}, \mathcal{X}_{\beta}\right) \psi_{\beta}\left(\mathcal{X}_{\beta}\right), \quad(\mathrm{A} 7 \mathrm{a}) \\
& S_{\text {eff,TE}}\left(\psi_{1}, \psi_{2}\right) \\
& =\frac{1}{2} \sum_{\alpha, \beta} \iint d \mathcal{X}_{\alpha} d \mathcal{X}_{\beta} \psi_{\alpha}\left(\mathcal{X}_{\alpha}\right)\left[\partial_{n_{\alpha}} \partial_{\mathrm{n}_{\beta}} \mathcal{G}\left(\mathcal{X}_{\alpha}, \mathcal{X}_{\beta}\right)\right] \psi_{\beta}\left(\mathcal{X}_{\beta}\right) .
\end{aligned}
$$

Here the Green function of the fields after Wick rotation is denoted by the new font $\mathcal{G}$. The partition functions defined by Eq. (A6) are calculated straightforwardly, and the results are given in Eqs. (20) and (21) in the main text.

\section{APPENDIX B: GREEN TENSOR FOR PLANAR MULTILAYER MAGNETODIELECTRIC MEDIA WITH GAIN}

For planar multilayer geometries as illustrated in Fig. 1, the electric permittivity and magnetic permeability vary only in the $z$ direction, so we may introduce a transverse spatial Fourier transform as

$$
\mathbf{G}_{\mathrm{EM}}\left(\mathbf{x}-\mathbf{x}^{\prime}, z, z^{\prime}, i \omega\right)=\int \mathrm{d}^{2} \mathbf{q} e^{i \mathbf{q} \cdot\left(\mathbf{x}-\mathbf{x}^{\prime}\right)} \mathbf{G}_{\mathrm{EM}}\left(\mathbf{q}, z, z^{\prime}, i \omega\right)
$$

where $\mathbf{q}$ is a vector parallel to the conductor. Tomaš uses this to arrive at the solution of Eq. (14) in lossy dielectric multilayers [58]. The generalization to lossy magnetodielectric media can be found in Refs. [70] and [65]. Here we briefly describe the results of a further nontrivial generalization, namely to magnetodielectrics with both loss and gain. In our notation we follow Ref. [58].

The Green tensor $\mathbf{G}_{\mathrm{EM}}\left(\mathbf{q}, z, z^{\prime}, i \omega\right)$ assumes two different forms, depending on whether $z$ and $z^{\prime}$ are located in the same layer. For $z^{\prime}$ in layer $j$, it is given by

$$
\begin{aligned}
& \mathbf{G}_{\mathrm{EM}}\left(\mathbf{q}, z, z^{\prime}, i \omega\right)=\frac{1}{\varepsilon_{0} \varepsilon_{j}(i \omega) \omega^{2}} \delta\left(z-z^{\prime}\right) \hat{z} \hat{z}+\frac{\mu_{0} \mu_{j}(i \omega)}{2 \mathcal{Q}_{j}} \\
& \times \sum_{\sigma=\mathrm{TE}}^{\mathrm{TM}} \xi_{\sigma} \frac{e^{-\mathcal{Q}_{j} d_{j}}}{D_{j}^{\sigma}} \\
& \times\left[\boldsymbol{\varepsilon}_{j}^{\sigma>}(\mathbf{q}, i \omega ; z) \boldsymbol{\varepsilon}_{j}^{\sigma<}\left(-\mathbf{q}, i \omega ; z^{\prime}\right) \Theta\left(z-z^{\prime}\right)\right. \\
& \left.+\boldsymbol{\varepsilon}_{j}^{\sigma<}(\mathbf{q}, i \omega ; z) \boldsymbol{\varepsilon}_{j}^{\sigma>}\left(-\mathbf{q}, i \omega ; z^{\prime}\right) \Theta\left(z^{\prime}-z\right)\right] \\
& \text { for } z \text { in layer } j \\
& \mathbf{G}_{\mathrm{EM}}\left(\mathbf{q}, z, z^{\prime}, i \omega\right)=\frac{\mu_{0} \mu_{l}(i \omega)}{2 \mathcal{Q}_{l}} \sum_{\sigma=\mathrm{TE}}^{\mathrm{TM}} \xi_{\sigma} \frac{t_{l j j}^{\sigma} e^{-\left(\mathcal{Q}_{j} d_{j}+\mathcal{Q}_{l} d_{l}\right)}}{D_{j}^{\sigma}} \\
& \times\left[\frac{\boldsymbol{\varepsilon}_{l}^{\sigma>}(\mathbf{q}, i \omega ; z)}{D_{l / j}^{+, \sigma}} \boldsymbol{\varepsilon}_{j}^{\sigma<}\left(-\mathbf{q}, i \omega ; z^{\prime}\right) \Theta(l-j)\right.
\end{aligned}
$$

$$
\begin{aligned}
& \left.+\frac{\boldsymbol{\varepsilon}_{l}^{\sigma<}(\mathbf{q}, i \omega ; z)}{D_{l / j}^{-, \sigma}} \boldsymbol{\varepsilon}_{j}^{\sigma>}\left(-\mathbf{q}, i \omega ; z^{\prime}\right) \Theta(j-l)\right] \\
& \text { for } z \text { in layer } l \neq j
\end{aligned}
$$

where $\xi_{\mathrm{TE}}=-1, \xi_{\mathrm{TM}}=1, \Theta(z)$ is the usual unit step function, and

$$
\begin{gathered}
\boldsymbol{\varepsilon}_{j}^{\sigma>}(\mathbf{q}, i \omega ; z)=\mathbf{e}_{\sigma j}^{+}(\mathbf{q}) e^{-\mathcal{Q}_{j}\left(z-d_{j}\right)}+r_{j^{+}}^{\sigma} \mathbf{e}_{\sigma j}^{-}(\mathbf{q}) e^{\mathcal{Q}_{j}\left(z-d_{j}\right)} \\
\boldsymbol{\varepsilon}_{j}^{\sigma<}(\mathbf{q}, i \omega ; z)=\mathbf{e}_{\sigma j}^{-}(\mathbf{q}) e^{\mathcal{Q}_{j} z}+r_{j}^{\sigma} \mathbf{e}_{\sigma j}^{+}(\mathbf{q}) e^{-\mathcal{Q}_{j} z} .
\end{gathered}
$$

Here $\sigma$ stands for TE or TM, and $\mathbf{e}_{\mathrm{TE} j}^{ \pm}=(\hat{\mathbf{q}} \times \hat{z})_{j}$ and $\mathbf{e}_{\mathrm{TM} j}^{ \pm}=$ $\frac{-1}{q_{j}}\left(i|\mathbf{q}| \hat{z} \pm \mathcal{Q}_{j} \hat{\mathbf{q}}\right)_{j}$ are the polarization vectors for TE and TM polarized waves propagating in the positive- $z$ and negative- $z$ directions, with $q_{j} \equiv \sqrt{\omega^{2} \varepsilon_{j}(i \omega) \mu_{j}(i \omega) / c^{2}}$ and

$$
\mathcal{Q}_{j}(\mathbf{q}, i \omega)=\sqrt{q^{2}+\omega^{2} \varepsilon_{j}(i \omega) \mu_{j}(i \omega) / c^{2}},
$$

which can be expressed in terms of the magnitude of the $z$ component $\kappa_{j}(\mathbf{q}, \omega)=\sqrt{\omega^{2} \varepsilon_{j}(\omega) \mu_{j}(\omega) / c^{2}-q^{2}}$ of the wave vector in layer $j$ as $\mathcal{Q}_{j}(\mathbf{q}, i \omega)=-i \kappa_{j}(i \omega)$.

Here we arrive at a subtlety in the determination of the Green tensor for active multilayer media: the $z$ component of the wave vector, $\kappa_{j}(\mathbf{q}, \omega)$, is not always well defined for real frequencies. The subtlety is that although the refractive index has no branch points in the upper half of the plane, $\kappa_{j}(\mathbf{q}, \omega)$ may have branch points there [60]. If there are such branch points, then $\kappa_{j}(\mathbf{q}, \omega)$ loses its usual physical interpretation. We follow Refs. [61] and [60] and only consider active media without branch points where $\kappa_{j}(\omega)$ is meaningful for real frequencies. In that case, the signs of $\operatorname{Re}\left[\kappa_{j}(\omega)\right]$ and $\operatorname{Im}\left[\kappa_{j}(\omega)\right]$ are identical to those of $\operatorname{Re}\left[n_{j}(\omega)\right]$ and $\operatorname{Im}\left[n_{j}(\omega)\right]$, respectively, where $n_{j}$ is refractive index of the $j$ th layer (see Refs. [61] and [60]). Other quantities in Eqs. (B2) that still need to be defined are

$$
\begin{aligned}
D_{j}^{\sigma} & =1-r_{j^{-}}^{\sigma} r_{j^{+}}^{\sigma} e^{-2 Q_{j} d_{j}}, \\
D_{l / j}^{ \pm, \sigma} & =1-r_{l \pm}^{\sigma} r_{l l \mp 1 / j}^{\sigma} e^{-2 Q_{l} d_{l}},
\end{aligned}
$$

where $r_{j^{-}}^{\sigma}\left(r_{j^{+}}^{\sigma}\right)$ are the generalized coefficients for reflection at the left (right) boundary of layer $j$, which can be calculated with the aid of the recursive relations $[65,70]$

$$
r_{j \pm}^{\mathrm{TE}}=\frac{\left(\frac{\mu_{j \pm 1}}{\mathcal{Q}_{j \pm 1}}-\frac{\mu_{j}}{\mathcal{Q}_{j}}\right)+\left(\frac{\mu_{j \pm 1}}{\mathcal{Q}_{j \pm 1}}+\frac{\mu_{j}}{\mathcal{Q}_{j}}\right) e^{-2 \mathcal{Q}_{j \pm 1} d_{j \pm 1}} r_{j \pm 1 \pm}^{\mathrm{TE}}}{\left(\frac{\mu_{j \pm 1}}{\mathcal{Q}_{j \pm 1}}+\frac{\mu_{j}}{\mathcal{Q}_{j}}\right)+\left(\frac{\mu_{j \pm 1}}{\mathcal{Q}_{j \pm 1}}-\frac{\mu_{j}}{\mathcal{Q}_{j}}\right) e^{-2 \mathcal{Q}_{j \pm 1} d_{j \pm 1}} r_{j \pm 1 \pm}^{\mathrm{TE}}}
$$

for TE-polarized light, and

$$
r_{j \pm}^{\mathrm{TM}}=\frac{\left(\frac{\varepsilon_{j \pm 1}}{\mathcal{Q}_{j \pm 1}}-\frac{\varepsilon_{j}}{\mathcal{Q}_{j}}\right)+\left(\frac{\varepsilon_{j \pm 1}}{\mathcal{Q}_{j \pm 1}}+\frac{\varepsilon_{j}}{\mathcal{Q}_{j}}\right) e^{-2 \mathcal{Q}_{j \pm 1} d_{j \pm 1}} r_{j \pm 1 \pm}^{\mathrm{TM}}}{\left(\frac{\varepsilon_{j \pm 1}}{\mathcal{Q}_{j \pm 1}}+\frac{\varepsilon_{j}}{\mathcal{Q}_{j}}\right)+\left(\frac{\varepsilon_{j \pm 1}}{\mathcal{Q}_{j \pm 1}}-\frac{\varepsilon_{j}}{\mathcal{Q}_{j}}\right) e^{-2 \mathcal{Q}_{j \pm 1} d_{j \pm 1}} r_{j \pm 1 \pm}^{\mathrm{TM}}}
$$

for TM polarization. For a finite number of layers, there is only a finite number of relations to be solved, since for the leftmost and rightmost layers one should take $r_{1-}^{\mathrm{TE}, \mathrm{TM}}=0$, $r_{n+}^{\mathrm{TE}, \mathrm{TM}}=0$, and $d_{1}=d_{n}=0$. From the definition of the Fresnel coefficients introduced, it follows that they satisfy

$$
r_{i / j / k}^{\sigma}=\frac{1}{D_{j}^{\sigma}}\left[r_{i / j}^{\sigma}+\left(t_{i / j}^{\sigma} t_{j / i}^{\sigma}-r_{i / j}^{\sigma} r_{j / i}^{\sigma}\right) r_{j / k}^{\sigma} e^{-2 \mathcal{Q}_{j} d_{j}}\right],
$$


in the notation of Ref. [58].

We have hereby specified the rather complicated expression for the Green tensor $\mathbf{G}$ of Eq. (B2), and we still need to relate it to the Green tensors $\mathcal{G}^{\mathrm{TE}, \mathrm{TM}}\left(\mathbf{q}, z, z^{\prime}, i \omega\right)$, in terms of which the Casimir force is expressed in Eq. (21). By using the ordinary coordinates according to the convention of Schwinger et al. [3] and choosing $(\hat{q}, \hat{z}, \hat{q} \times \hat{z}) \longrightarrow(\hat{x}, \hat{z},-\hat{y})$ [8], the transverse electric and transverse magnetic Green functions satisfy

$$
\begin{aligned}
& {\left[-\frac{\partial}{\partial z} \frac{1}{\mu(i \omega, z)} \frac{\partial}{\partial z}+\frac{q^{2}}{\mu(i \omega, z)}+\frac{\omega^{2} \varepsilon(i \omega, z)}{c^{2}}\right] \mathcal{G}^{\mathrm{TE}}\left(\mathbf{q}, z, z^{\prime}, i \omega\right),} \\
& \quad=\mu_{0} \delta\left(z-z^{\prime}\right), \\
& {\left[-\frac{\partial}{\partial z} \frac{1}{\varepsilon(i \omega, z)} \frac{\partial}{\partial z}+\frac{q^{2}}{\varepsilon(i \omega, z)}+\frac{\omega^{2} \mu(i \omega, z)}{c^{2}}\right] \mathcal{G}^{\mathrm{TM}}\left(\mathbf{q}, z, z^{\prime}, i \omega\right)} \\
& =\mu_{0} \delta\left(z-z^{\prime}\right) .
\end{aligned}
$$

We checked with some lengthy but straightforward calculations that these Green functions $\mathcal{G}^{\mathrm{TE}, \mathrm{TM}}\left(\mathbf{q}, z, z^{\prime}, i \omega\right)$ with $z^{\prime}$ and $z$ in layers $j$ and $l$ respectively can be written very elegantly in terms of $\mathbf{G}\left(\mathbf{q}, z, z^{\prime}, i \omega\right)$ as

$$
\begin{aligned}
& \mathbf{G}_{y y}\left(\mathbf{q}, z, z^{\prime}, i \omega\right)=\mathcal{G}^{\mathrm{TE}}\left(\mathbf{q}, z, z^{\prime}, i \omega\right), \\
& \mathbf{G}_{z z}\left(\mathbf{q}, z, z^{\prime}, i \omega\right) \\
& =\delta_{l j} \frac{\delta\left(z-z^{\prime}\right)}{\varepsilon_{0} \varepsilon(i \omega, z) \omega^{2}}+\frac{q^{2} c^{2}}{\varepsilon(i \omega, z) \varepsilon\left(i \omega, z^{\prime}\right) \omega^{2}} \\
& \quad \times \mathcal{G}^{\mathrm{TM}}\left(\mathbf{q}, z, z^{\prime}, i \omega\right) .
\end{aligned}
$$

It is important to point out what has been achieved here: By the identifications (B9) we have found solutions for the scalar Green functions $\mathcal{G}^{\mathrm{TE}}$,TM that are defined by the equations (B8), with boundary conditions that follow from the continuity of $H_{x}, H_{y}$, and $\mu H_{z}$, and of $E_{x}, E_{y}$, and $\varepsilon E_{z}$.
[1] H. B. G. Casimir, Proc. K. Ned. Akad. Wet. 51, 793 (1948).

[2] E. M. Lifshitz, Zh. Eksp. Teor. Fiz. 29, 94 (1956) [Sov. Phys. JETP 2, 73 (1956)]; I. D. Dzyaloshinskii, E. M. Lifshitz, and L. P. Pitaevskii, Usp. Fiz. Nauk 73, 381 (1961) [Sov. Phys. Usp. 4, 153 (1961)].

[3] J. Schwinger, L. L. DeRaad Jr., and K. A. Milton, Ann. Phys. (NY) 115, 1 (1978).

[4] F. Zhou and L. Spruch, Phys. Rev. A 52, 297 (1995).

[5] G. L. Klimchitskaya, U. Mohideen, and V. M. Mostepanenko, Phys. Rev. A 61, 062107 (2000).

[6] M. S. Tomaš, Phys. Rev. A 66, 052103 (2002); Phys. Lett. A 342, 381 (2005).

[7] C. Raabe, L. Knoll, and D.-G. Welsch, Phys. Rev. A 68, 033810 (2003); C. Raabe and D.-G. Welsch, ibid. 71, 013814 (2005).

[8] S. A. Ellingsen, J. Phys. A 40, 1951 (2007); S. A. Ellingsen and I. Brevik, J. Phys. A: Math. Theor. 40, 3643 (2007).

[9] H. B. Chan, V. A. Aksyuk, R. N. Kleiman, D. J. Bishop, and F. Capasso, Phys. Rev. Lett. 87, 211801 (2001).

[10] H. B. Chan, V. A. Aksyuk, R. N. Kleiman, D. J. Bishop, and F. Capasso, Science 291, 1941 (2001).

[11] J. N. Munday, F. Capasso, and V. A. Parsegian, Nature (London) 457, 170 (2009).

[12] M. J. Spaarnaay, Physica 24, 751 (1958).

[13] E. S. Sabisky and C. H. Anderson, Phys. Rev. A 7, 790 (1973).

[14] S. K. Lamoreaux, Phys. Rev. Lett. 78, 5 (1997); 81, 5475 (1998).

[15] U. Mohideen and A. Roy, Phys. Rev. Lett. 81, 4549 (1998); A. Roy and U. Mohideen, ibid. 82, 4380 (1999).

[16] G. Bressi, G. Carugno, R. Onofrio, and G. Ruoso, Phys. Rev. Lett. 88, 041804 (2002).

[17] H. B. Chan, Y. Bao, J. Zou, R. A. Cirelli, F. Klemens, W. M. Mansfield, and C. S. Pai, Phys. Rev. Lett. 101, 030401 (2008).

[18] C. Hertlein, L. Helden, A. Gambassi, S. Dietrich, and C. Bechinger, Nature (London) 451, 172 (2008).

[19] W. J. Kim, A. O. Sushkov, D. A. R. Dalvit, and S. K. Lamoreaux, Phys. Rev. Lett. 103, 060401 (2009).

[20] S. de Man, K. Heeck, R. J. Wijngaarden, and D. Iannuzzi, Phys. Rev. Lett. 103, 040402 (2009).
[21] M. Masuda and M. Sasaki, Phys. Rev. Lett. 102, 171101 (2009)

[22] H.-J. Butt et al., Surf. Sci. Rep. 59, 1 (2005).

[23] F. Capasso, J. N. Munday, D. Iannuzzi, and H. B. Chan, IEEE J. Sel. Top. Quantum Electron. 13, 400 (2007).

[24] D. Iannuzzi, J. Munday, and F. Capasso, US Patent No. 20,070,066,494 (pending).

[25] A. A. Feiler, L. Bergstrom, and M. W. Rutland, Langmuir 24, 2274 (2008).

[26] F. S. S. Rosa, D. A. R. Dalvit, and P. W. Milonni, Phys. Rev. Lett. 100, 183602 (2008).

[27] S. K. Lamoreaux, Nature (London) 457, 156 (2009).

[28] I. G. Pirozhenko and A. Lambrecht, J. Phys. A: Math. Theor. 41, 164015 (2008).

[29] R. Zhao, J. Zhou, Th. Koschny, E. N. Economou, and C. M. Soukoulis, Phys. Rev. Lett. 103, 103602 (2009); R. Zhao, Th. Koschny, E. N. Economou, and C. M. Soukoulis, Phys. Rev. B 81, 235126 (2010).

[30] T. H. Boyer, Phys. Rev. A 9, 2078 (1974).

[31] O. Kenneth, I. Klich, A. Mann, and M. Revzen, Phys. Rev. Lett. 89, 033001 (2002).

[32] Y. Sherkunov, Phys. Rev. A 72, 052703 (2005).

[33] U. Leonhardt and T. G. Philbin, New J. Phys. 9, 254 (2007).

[34] R. J. Glauber, in Frontiers in Quantum Optics, edited by E. R. Pike and S. Sarkar (Hilger, Bristol, 1986).

[35] J. R. Jeffers, N. Imoto, and R. Loudon, Phys. Rev. 47, 3346 (1993).

[36] J. Jeffers, S. M. Barnett, R. Loudon, R. Matloob, and M. Artoni, Opt. Commun. 131, 66 (1996).

[37] R. Matloob, R. Loudon, M. Artoni, S. M. Barnett, and J. Jeffers, Phys. Rev. A 55, 1623 (1997).

[38] S. Scheel, L. Knöll, and D.-G. Welsch, Phys. Rev. A 58, 700 (1998).

[39] C. Raabe and D.-G. Welsch, Eur. Phys. J. Spec. Top. 160, 371 (2008).

[40] A. Sambale, S. Y. Buhmann, H. T. Dung, and D.-G. Welsch, Phys. Rev. A 80, 051801(R) (2009). 
[41] A. Sambale, D.-G. Welsch, S. Y. Buhmann, and H. T. Dung, Opt. Spectr. 108, 391 (2010).

[42] C. Raabe and D.-G. Welsch, Phys. Rev. A 71, 013814 (2005).

[43] L. P. Pitaevskii, Phys. Rev. A 73, 047801 (2006).

[44] I. Brevik and S. A. Ellingsen, Phys. Rev. A 79, 027801 (2009).

[45] H. Li and M. Kardar, Phys. Rev. Lett. 67, 3275 (1991); Phys. Rev. A 46, 6490 (1992).

[46] R. Golestanian and M. Kardar, Phys. Rev. A 58, 1713 (1998).

[47] T. Emig, A. Hanke, R. Golestanian, and M. Kardar, Phys. Rev. Lett. 87, 260402 (2001); Phys. Rev. A 67, 022114 (2003).

[48] F. Kheirandish, M. Soltani, and J. Sarabadani, Phys. Rev. A 81, 052110 (2010).

[49] B. Huttner and S. M. Barnett, Phys. Rev. A 46, 4306 (1992); Europhys. Lett. 18, 487 (1992).

[50] M. Wubs and L. G. Suttorp, Phys. Rev. A 63, 043809 (2001).

[51] L. G. Suttorp and M. Wubs, Phys. Rev. A 70, 013816 (2004).

[52] F. Kheirandish and M. Amooshahi, Phys. Rev. A 74, 042102 (2006).

[53] F. Kheirandish and M. Soltani, Phys. Rev. A 78, 012102 (2008).

[54] F. Kheirandish and E. Amooghorban, Phys. Rev. A 82, 042901 (2010).

[55] T. G. Philbin, New J. Phys. 12, 123008 (2010).
[56] L. Ryder, Quantum Field Theory, 2nd ed. (Cambridge University Press, Cambridge, 1996).

[57] D. C. Burnham and R. Y. Chiao, Phys. Rev. 188, 667 (1969); R. Y. Chiao, Phys. Rev. A 48, R34 (1993).

[58] M. S. Tomaš, Phys. Rev. A 51, 2545 (1995).

[59] L. D. Landau and E. M. Lifschitz, Statistical Physics Part I, 3rd ed. (Pergamon, Oxford, 1980), Sec. 123.

[60] J. Skaar, Opt. Lett. 31, 3372 (2005).

[61] J. Skaar, Phys. Rev. E 73, 026605 (2006); B. Nistad and J. Skaar, ibid. 78, 036603 (2008);

[62] A. Akyurtlu and A.-G. Kussow, Phys. Rev. A 82, 055802 (2010).

[63] M. Schaden, L. Spruch, and F. Zhou, Phys. Rev. A 57, 1108 (1998).

[64] A. Lambrecht, M.-T. Jaekel, and S. Reynaud, Phys. Lett. A 225 , 188 (1997).

[65] S. Y. Buhmann, D.-G. Welsch, and T. Kampf, Phys. Rev. A 72, 032112 (2005)

[66] L. P. Teo, Phys. Rev. A 81, 032502 (2010).

[67] J. D. Jackson, Classical Electrodynamics (New York, Wiley, 1998).

[68] F. Kheirandish, E. Amooghorban, and M. Soltani, Phys. Rev. A 83, 032507 (2011).

[69] M. Bordag, G. L. Klimchitskaya, U. Mohideen, and V. M. Mostepanenko, Advances in the Casimir Effect (Oxford University Press, Oxford, 2009).

[70] W. C. Chew, Waves and Fields in Inhomogeneous Media (IEEE, New York, 1995). 